ESAIM: M2AN

Vol. 40, No 4, 2006, pp. 785-814

DOI: $10.1051 / \mathrm{m} 2 \mathrm{an}: 2006030$
ESAIM: Mathematical Modelling and Numerical Analysis

www.edpsciences.org/m2an

\title{
FINITE ELEMENT ANALYSIS OF A SIMPLIFIED STOCHASTIC HOOKEAN DUMBBELLS MODEL ARISING FROM VISCOELASTIC FLOWS*
}

\author{
Andrea Bonito $^{1}$, Philippe Clément $^{2}$ and Marco PiCasso ${ }^{1}$
}

\begin{abstract}
A simplified stochastic Hookean dumbbells model arising from viscoelastic flows is considered, the convective terms being disregarded. A finite element discretization in space is proposed. Existence of the numerical solution is proved for small data, so as a priori error estimates, using an implicit function theorem and regularity results obtained in [Bonito et al., J. Evol. Equ. 6 (2006) 381-398] for the solution of the continuous problem. A posteriori error estimates are also derived. Numerical results with small time steps and a large number of realizations confirm the convergence rate with respect to the mesh size.
\end{abstract}

Mathematics Subject Classification. 46T, 65M, 76A.

Received: December 2, 2005. Revised: May 1st, 2006.

\section{INTRODUCTION}

Numerical modeling of viscoelastic flows is of great importance for complex engineering applications involving blood, paints or adhesives. In the traditional macroscopic approach the unknowns are the velocity, the pressure and the extra-stress satisfying the mass and momentum equations supplemented with a so-called constitutive equation. This constitutive equation between the velocity and the stress can be either differential or integral $[9,57]$.

The simplest macroscopic example is the Oldroyd-B model which can be derived from the mesoscopic Hookean dumbbells model. The stochastic dumbbells model corresponds to a dilute solution of liquid polymer, that is a newtonian solvent with non interacting polymer chains. The polymer chains are modeled by dumbbells, two beads connected with elastic springs, see Figure 1.

\footnotetext{
Keywords and phrases. Viscoelastic, Hookean dumbbells, finite elements, stochastic differential equations.

* A.B. is supported by the Swiss National Science Foundation.

1 Institut d'Analyse et Calcul Scientifique, École Polytechnique Fédérale de Lausanne, 1015 Lausanne, Switzerland. andrea.bonito@a3.epfl.ch, marco.picasso@epfl.ch

2 Mathematical Institute, Leiden University, P.O. Box 9512, NL-2300 RA Leiden, The Netherlands.

PPJEClement@netscape.net
} 


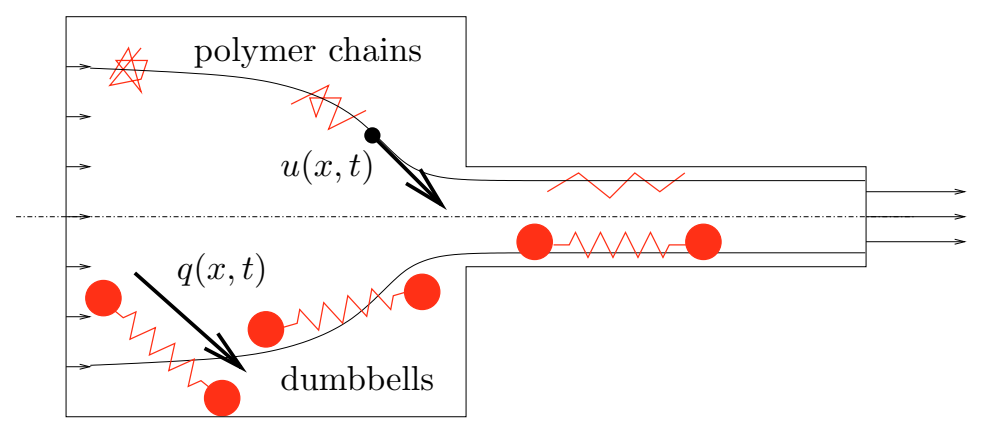

FigurE 1. The mesoscopic dumbbells model for a dilute solution of liquid polymer.

The mass and momentum conservation laws lead to the following partial differential equations for the velocity $u$, the pressure $p$ and the extra-stress $\sigma$

$$
\begin{aligned}
\rho\left(\frac{\partial u}{\partial t}+(u \cdot \nabla) u\right)-\nabla \cdot\left(2 \eta_{s} \epsilon(u)+\sigma\right)+\nabla p & =f \\
\nabla \cdot u & =0 .
\end{aligned}
$$

Here $\rho$ is the density, $f$ a force term, $\eta_{s}$ is the solvent viscosity and $\epsilon(u)=\frac{1}{2}\left(\nabla u+(\nabla u)^{T}\right)$ is the symmetric part of the velocity gradient. On the other hand, the dimensionless spring elongation $q$ satisfies the following stochastic differential equation

$$
\left.\mathrm{d} q=(-(u \cdot \nabla) q)+(\nabla u) q-\frac{1}{2 \lambda} \mathrm{F}(q)\right) \mathrm{d} t+\frac{1}{\sqrt{\lambda}} \mathrm{d} B,
$$

where $\lambda$ is the relaxation time, $\mathrm{F}$ is the force due to the elastic spring and $B$ is a vector of independent Wiener processes modeling the thermal agitation and collisions with the solvent molecules. The transport term $(u \cdot \nabla) q$ in (0.3) corresponds to the fact that the trajectories of the dumbbells center of mass are those of the liquid particles. The term $(\nabla u) q$ takes into account the drag force due to the beads. The extra-stress $\sigma$ is then obtained by the mean of the closure equation

$$
\sigma=\frac{\eta_{p}}{\lambda}(\mathbb{E}(q \otimes \mathrm{F}(q))-I)
$$

with $\eta_{p}$ the polymer viscosity. The case $\mathrm{F}(q)=q$, namely Hookean dumbbells, leads to the Oldroyd-B model where the extra-stress $\sigma$ satisfies

$$
\sigma+\lambda\left(\frac{\partial \sigma}{\partial t}+(u \cdot \nabla) \sigma-(\nabla u) \sigma-\sigma(\nabla u)^{T}\right)=2 \eta_{p} \epsilon(u)
$$

The FENE dumbbells model (see $[9,57]$ for a detailed description) is a more realistic model corresponding to $F(q)=\frac{q}{1-q^{2} / b}$, where $b>0$ depends on the number of monomer units of a polymer chain. The goal of the FENE model is to take into account the finite extensibility of the polymer chains. In that case, there is no equivalent constitutive relation for the extra-stress, but closure approximations (such as FENE-P, see for instance $[9,57]$ ) have been derived. These approximations can have significant impact on rheological prediction, see for instance $[1,17,43]$. Recently, due to increasing computational resources, equations $(0.1)-(0.4)$ have been solved numerically to obtain more realistic results $[14,16,18,43,46,47]$. For a review of numerical methods used in viscoelastic flows we refer for instance to $[3,44,58]$. 
The kinetic theory can also be formulated by introducing the probability density $f(x, q, t)$ of the spring elongation which must satisfy a Fokker-Planck equation. We refer to $[21,29,30]$ for numerical experiments and $[8,62]$ for a mathematical analysis. This deterministic approach seems to be inappropriate when considering more complex kinetic models involving chains [44], although recent advances are encouraging [68].

We will focus in this paper on the stochastic description of the simplest dumbbells model, namely the Hookean dumbbells model $F(q)=q$. Although the Hookean dumbbells model is too simple to reproduce experiments such as shear thinning for instance, it already contains some numerical difficulties included in the kinetic theory. At the continuous level, the model is formally equivalent to the Oldroyd-B model but the equivalence does not hold when considering equal order finite element discretizations. Thus, to the major difficulties already present in the macroscopic model, we must add those coming from stochastic modeling. All these difficulties gathered are:

(i) the presence of the quadratic term $(\nabla u) q$ which prevents to obtain a priori estimates leading to existence and convergence for any data;

(ii) the presence of the convective term $(u \cdot \nabla) q$ which requires an adequate mathematical analysis [48] and the use of numerical schemes suited to transport dominated problems;

(iii) the case $\eta_{s}=0$ which require either a compatibility condition between the finite element spaces for $u$, $q$ and $p$ or the use of adequate stabilization procedures, such as EVSS for instance;

(iv) the Wiener process in (0.3) which requires efficient procedures such as variance reduction to be considered, see for instance $[13,18,39,44]$.

Concerning the analysis and numerical analysis of macroscopic viscoelastic models, a large amount of publications can be found. The existence of slow steady viscoelastic flow has been proved in $[2,61]$. For the time-dependent case, existence of solutions locally in time and, for small data, globally in time has been proved in [37] in Hilbert spaces. Extensions to Banach spaces and a review can be found in [32]. Finally, existence for any data has been proved in [52] for a corotational Oldroyd model only. Convergence of finite element methods for the linear three fields Stokes problem have been studied for instance in $[15,33,34,64]$. Convergence of continuous and discontinuous finite element methods for steady state viscoelastic fluids have been presented in $[6,31,56,65]$, provided the solution of the continuous problem is smooth and small enough. Extension to time-dependent problems have been proposed in $[7,27,28,55]$.

On the other hand, few papers pertaining to the kinetic theory have been published. From the analysis point of view, the deterministic (Fokker-Planck) formulation has been studied in $[8,51,62,69]$. Concerning the stochastic formulation, existence of FENE dumbbells in one space dimension is proved in [40], long-time asymptotics are used in [41]. The well posedeness of the dumbbells model in three space dimensions has been proved for nonlinear elastic dumbbells in [26].

The complete analysis and numerical analysis of a one dimensional Hookean dumbbells shear flows can be found in [38]. The authors consider the error due to space and time discretization, but also the error due to the Monte Carlo method. Optimal convergence is obtained for the velocity in the $L^{2}\left(H^{1}\right)$ norm (the $L^{2}\left(L^{2}\right)$ norm is considered in [49]), a similar study is available in [25]. From the authors knowledge, the only numerical analysis in more than one space dimension is [50]. An implicit finite difference method is considered in the unit square (or cube) with periodic boundary conditions. Assuming the velocity $u \in \mathcal{C}^{5}$ and the time step $\tau=O\left(h^{2}\right)$, the authors prove optimal convergence rates.

The numerical analysis of a finite element method in more than one space dimension is still missing. In this paper, only the finite element discretization in space is considered and the numerical analysis is proposed for a simplified time-dependent Hookean dumbbells problem in dimension two. More precisely, we disregard items (ii) and (iii) above, assume $\eta_{s}>0$ and remove the convective terms. The reason for removing the convective terms is motivated by the fact that this simplified problem corresponds to the correction step in the splitting algorithm described in $[12,36]$ for solving viscoelastic flows with complex free surfaces. The consequence when removing convective terms is that the implicit function theorem can be used to prove convergence results, whenever the data are small enough, using the same techniques as in [10,59]. Existence and regularity has already been proved in [11], this regularity being sufficient to prove convergence of a finite element discretization in space. 
The outline of the paper is as follows. The continuous problem and its finite elements scheme are described in the next section. Then, some notations and the results of [11] are presented. Existence of the finite element solution and a priori error estimates are established in Section 3. A posteriori error estimates are derived in Section 4. Finally, numerical results with small time steps and a large number of realizations confirm the convergence rate with respect to the mesh size.

\section{The simplified Hookean dumbbells problem and its finite Element APPROXIMATION IN SPACE}

Let $D$ be a bounded, connected open set of $\mathbb{R}^{d}, d=2$ or 3 with boundary $\partial D$ of class $\mathcal{C}^{2}$, and let $T>0$. Let $(\Omega, \mathcal{F}, \mathcal{P})$ be a complete filtered probability space. The filtration $\mathcal{F}_{t}$ upon which the Brownian process $B$ is defined is completed with respect to $\mathcal{P}$ and is assumed to be right continuous on $[0, T]$.

Given the initial conditions $q_{0}: \Omega \rightarrow \mathbb{R}^{d}, u_{0}: D \rightarrow \mathbb{R}^{d}$, a force term $f$, constant solvent and polymer viscosities $\eta_{s}>0, \eta_{p}>0$, a constant relaxation time $\lambda>0$, we are searching for the velocity $u: D \times(0, T) \rightarrow \mathbb{R}^{d}$, the pressure $p: D \times(0, T) \rightarrow \mathbb{R}$ and the elongation vector $q: D \times(0, T) \times \Omega \rightarrow \mathbb{R}^{d}$, which must satisfy

$$
\begin{array}{ll}
\mathrm{d} q-\left((\nabla u) q-\frac{1}{2 \lambda} q\right) \mathrm{d} t-\frac{1}{\sqrt{\lambda}} \mathrm{d} B=0 & \text { in } D \times(0, T) \times \Omega, \\
\rho \frac{\partial u}{\partial t}-\nabla \cdot\left(2 \eta_{s} \epsilon(u)+\frac{\eta_{p}}{\lambda}(\mathbb{E}(q \otimes q)-I)\right)+\nabla p=f & \text { in } D \times(0, T), \\
\nabla \cdot u=0 & \text { in } D \times(0, T), \\
u(., 0)=u_{0} & \text { in } D, \\
q(., 0, .)=q_{0} & \text { in } D \times \Omega, \\
u=0 & \text { on } \partial D \times(0, T) .
\end{array}
$$

Remark 1.1. Equations (1.1) and (1.5) are notations for

$$
q(x, t, \omega)-q_{0}(t, \omega)-\int_{0}^{t}\left((\nabla u(x, s)) q(x, s, \omega)-\frac{1}{2 \lambda} q(x, s, \omega)\right) \mathrm{d} s-\frac{1}{\sqrt{\lambda}} B(t, \omega)=0
$$

where $(x, t, \omega) \in D \times[0, T] \times \Omega$.

Due to the regularity of the Brownian process, little Hölder spaces will be used. They are closed subset of the classical Hölder spaces $\mathcal{C}^{\mu}([0, T] ; E)$ and are defined for all Banach space $E$ and for all $0<\mu<1$ by

$$
h^{\mu}([0, T] ; E)=\left\{f \in \mathcal{C}^{\mu}([0, T] ; E) ; \lim _{\delta \rightarrow 0} \sup _{t, s \in[0, T],|t-s|<\delta} \frac{\|f(t)-f(s)\|_{E}}{|t-s|^{\mu}}=0\right\} .
$$

Provided with the norm of $\mathcal{C}^{\mu}([0, T] ; E)$, little Hölder spaces are Banach spaces and are separable Banach spaces assuming $E$ is a separable Banach space, see for instance [54] for more details. We will use the notation $h_{0}^{\mu}([0, T] ; E)$ for the restriction of functions of $h^{\mu}([0, T] ; E)$ vanishing at the origin. For simplicity, the notation will be abridged as follows whenever there is no possible confusion. For $d<r<\infty$, the space $L^{r}$ denotes $L^{r}(D ; \mathbb{R})$ or $L^{r}\left(D ; \mathbb{R}^{d}\right)$. Also, for $0<\mu<1 / 2$ and $2 \leq \gamma<\infty, h^{\mu}\left(L^{r}\right)$ stands for $h^{\mu}\left([0, T] ; L^{r}(D ; \mathbb{R})\right)$ or $h^{\mu}\left([0, T] ; L^{r}\left(D ; \mathbb{R}^{d}\right)\right)$ and $L^{\gamma}\left(h^{\mu}\left(L^{r}\right)\right)$ for $L^{\gamma}\left(\Omega ; h^{\mu}\left([0, T] ; L^{r}(D ; \mathbb{R})\right)\right)$ or $L^{\gamma}\left(\Omega ; h^{\mu}\left([0, T] ; L^{r}\left(D ; \mathbb{R}^{d}\right)\right)\right)$. The same notation applies for higher order spaces such as $W^{1, r}, h^{1+\mu}\left(W^{1, r}\right)$ and $L^{\gamma}\left(h^{1+\mu}\left(W^{1, r}\right)\right)$.

The implicit function theorem has been used in [11] to prove that (1.1)-(1.6) admits a unique solution

$$
u \in h^{1+\mu}\left(L^{r}\right) \cap h^{\mu}\left(W^{2, r}\right), \quad p \in h^{\mu}\left(W^{1, r} \cap L_{0}^{r}\right), \quad q \in L^{\gamma}\left(h^{\mu}\left(W^{1, r}\right)\right),
$$


with $2 \leq \gamma<\infty, d<r<\infty$ and $0<\mu<1 / 2$, for any data $\left(f, u_{0}\right)$ small enough in appropriate spaces and assuming the space $\Omega$ is rich enough to accommodate a given random vector $q_{0} \in L^{\gamma}(\Omega)$ such that

$$
\left\{\begin{array}{l}
q_{0} \text { is independent of } B \text { and }\left(q_{0}\right)_{i} \text { is independent of }\left(q_{0}\right)_{j}, 1 \leq i \neq j \leq d, \\
\text { and } \mathbb{E}\left(q_{0}\right)=0, \mathbb{E}\left(q_{0} \otimes q_{0}\right)=I .
\end{array}\right.
$$

Since $h^{\mu}\left([0, T] ; W^{1, r}(D)\right) \subset \mathcal{C}([0, T] \times \bar{D})$, let us notice that in particular, a process $q \in L^{\gamma}\left(h^{\mu}\left(W^{1, r}\right)\right)$ has a continuous sample path for almost each realization $\omega \in \Omega$.

In this paper we assume that the above existence result still holds when $D$ is a convex polygon in $\mathbb{R}^{2}$. The key point to prove this result when $D$ is a convex polygon is to prove that the negative Stokes operator $-A_{r}$ is still the generator of an analytic semi-group, see for instance [35]. We did not find such a result in the literature, therefore we will make this assumption and prove convergence of the finite element scheme. It should be noted that the corresponding property is true in stationary case for some $r>2$ depending on the angles of the polygon, see [59].

Let us introduce the finite element approximation in space for $D$, a convex polygon in $\mathbb{R}^{2}$. For any $h>0$, let $\mathcal{T}_{h}$ be a decomposition of $D$ into triangles $K$ with diameter $h_{K}$ less than $h$, regular in the sense of [22]. We consider $V_{h}, R_{h}$ and $Q_{h}$ the finite element spaces for the velocity, dumbbells elongation and pressure, respectively defined by:

$$
\begin{aligned}
V_{h} & =\left\{v_{h} \in \mathcal{C}^{0}\left(\bar{D} ; \mathbb{R}^{d}\right) ;\left.v_{h}\right|_{K} \in\left(\mathbb{P}_{1}\right)^{d} \quad \forall K \in \mathcal{T}_{h}\right\} \cap H_{0}^{1}\left(D ; \mathbb{R}^{d}\right), \\
R_{h} & =\left\{r_{h} \in \mathcal{C}^{0}\left(\bar{D} ; \mathbb{R}^{d}\right) ;\left.r_{h}\right|_{K} \in\left(\mathbb{P}_{1}\right)^{d} \quad \forall K \in \mathcal{T}_{h}\right\} \\
Q_{h} & =\left\{s_{h} \in \mathcal{C}^{0}(\bar{D} ; \mathbb{R}) ;\left.s_{h}\right|_{K} \in \mathbb{P}_{1} \quad \forall K \in \mathcal{T}_{h}\right\} \cap L_{0}^{2}(D ; \mathbb{R})
\end{aligned}
$$

We denote $i_{h}$ the $L^{2}(D)$ projection onto $V_{h}, R_{h}$ or $Q_{h}$ and introduce the following stabilized finite element discretization in space of (1.1)-(1.6). Given $f, u_{0}, q_{0}$ find

$$
\begin{aligned}
\left(u_{h}, q_{h}, p_{h}\right): \Omega \times(0, T) & \longrightarrow V_{h} \times R_{h} \times Q_{h}, \\
(\omega, t) & \longmapsto\left(u_{h}(t), q_{h}(\omega, t), p_{h}(t)\right),
\end{aligned}
$$

such that $u_{h}(0)=i_{h} u_{0}, q_{h}(0)=q_{0}$ and such that the following weak formulation holds in $(0, T) \times \Omega$ :

$$
\begin{aligned}
\rho\left(\frac{\partial u_{h}}{\partial t}, v_{h}\right)+2 \eta_{s}\left(\epsilon\left(u_{h}\right), \epsilon\left(v_{h}\right)\right)-\left(p_{h},\right. & \left.\nabla \cdot v_{h}\right)+\frac{\eta_{p}}{\lambda}\left(\mathbb{E}\left(q_{h} \otimes q_{h}\right)-I, \epsilon\left(v_{h}\right)\right)-\left(f, v_{h}\right) \\
+\left(\nabla \cdot u_{h}, s_{h}\right)+ & \sum_{K \in \mathcal{T}_{h}} \frac{\alpha h_{K}^{2}}{2 \eta_{p}}\left(\nabla p_{h}, \nabla s_{h}\right)_{K}+\left(q_{h}(t), r_{h}\right)-\left(1, r_{h}\right) q_{0} \\
& +\left(\int_{0}^{t}\left(\frac{1}{2 \lambda} q_{h}(k)-\left(\nabla u_{h}(k)\right) q_{h}(k)\right) \mathrm{d} k, r_{h}\right)-\frac{1}{\sqrt{\lambda}}\left(1, r_{h}\right) B=0,
\end{aligned}
$$

for all $\left(v_{h}, r_{h}, s_{h}\right) \in V_{h} \times R_{h} \times Q_{h}$. Here $\alpha>0$ is a dimensionless stabilization parameter and $(\cdot, \cdot)$ (respectively $(\cdot, \cdot)_{K}$ ) denotes the $L^{2}(D)$ (resp. $\left.L^{2}(K)\right)$ scalar product for scalars, vectors and tensors.

The main results of this paper are Theorems 3.6 and 4.2. Assuming the data $f$ and $u_{0}$ to be sufficiently small in an appropriate space (the space $Y$ defined in the next section), assuming the mesh size $h$ to be small enough, Theorem 3.6 states the existence of $\left(u_{h}, q_{h}\right) \in L^{2}\left(V_{h}\right) \times L^{2}\left(L^{\infty}\left(R_{h}\right)\right)$ solution of (1.9), unique in the neighbourhood of $(u, q)$, the solution of the continuous problem (1.1)-(1.6). Moreover, optimal a priori error estimates hold in the $L^{2}\left(H^{1}\right)$ norm for the velocity and in the $L^{2}\left(L^{\infty}\left(L^{2}\right)\right)$ norm for the dumbbells elongation. A posteriori error estimates are then proposed in Theorem 4.2. More precisely, assuming $f, u_{0}$ and $h$ to be sufficiently small, the error is bounded above by an explicit, residual based error estimator. 
The difficulty in proving such results is due to the fact that no useful (so far) a priori estimates are available due to the nonlinear term $\left(\nabla u_{h}\right) q_{h}$. The interested reader should note that an $L^{1}(D)$ estimate for the extrastress has been proved in [53] but it is not sufficent to prove convergence of finite element schemes. We will proceed as in the continuous problem [11]. More precisely, we will prove that the linearized problem in the neighborhood of the equilibrium state $u_{h}=0, q_{h}=q^{S}$ is well posed ( $q^{S}$ will be defined in the next section). Then, using an implicit function theorem taken from [20], existence and a priori error estimates will be obtained.

The above nonlinear finite element scheme is closely linked to the Oldroyd-B scheme studied in a previous paper [10]. However, the numerical schemes are not equivalent, therefore the analysis has to be done again. Moreover, it should be noted that in this paper the case $\eta_{s}=0$ is not considered, therefore some of the stabilization terms present in [14] are not included in the finite element formulation (1.9).

\section{Preliminaries on the COntinuous problem}

In this section, notations and results from [11] are recalled to the reader.

The proof of the existence of a solution $(u, q, p)$ satisfying (1.1)-(1.6) with the regularity (1.7) is based on the splitting

$$
q=q^{S}+q^{D} .
$$

The equilibrium state $q^{S}$ is the Ornstein-Uhlenbeck stochastic process independent of the space variable $x \in D$ which satisfies

$$
\mathrm{d} q^{S}=-\frac{1}{2 \lambda} q^{S} \mathrm{~d} t+\frac{1}{\sqrt{\lambda}} \mathrm{d} B, \quad q^{S}(0)=q_{0},
$$

whilst $q^{D}$ is the discrepancy with respect to the equilibrium $q^{S}$. The unknown function $q^{D}$ satisfies a differential equation with a stochastic forcing term

$$
\frac{\partial q^{D}}{\partial t}-\nabla u\left(q^{D}+q^{S}\right)+\frac{1}{2 \lambda} q^{D}=0, \quad q(0)=0 .
$$

Let $\left(X,\|\cdot\|_{X}\right)$ be the Banach space defined by

$$
X=\left\{(u, q) \in h^{1+\mu}\left(L^{r}\right) \cap h^{\mu}\left(W^{2, r}\right) \times L^{\gamma}\left(h^{1+\mu}\left(W^{1, r}\right) \cap h_{0}^{\mu}\left(W^{1, r}\right)\right) ; q \text { adapted to }\left(\mathcal{F}_{t}\right)_{t \in[0, T]}\right\},
$$

and let $\|\cdot\|_{X}$ be the product norm. Since existence (and uniqueness) of $q^{S} \in L^{\gamma}\left(\Omega ; h^{\mu}([0, T])\right.$ ) (see App. C) is ensured by classical results on stochastic differential equations, existence and uniqueness of problem (1.1)-(1.6) for small data arise from existence and uniqueness of $\left(u, q^{D}, p\right) \in X \times h^{\mu}\left(W^{1, r} \cap L_{0}^{r}\right)$ solution of

$$
\begin{array}{ll}
\frac{\partial q^{D}}{\partial t}-\nabla u\left(q^{D}+q^{S}\right)+\frac{1}{2 \lambda} q^{D}=0 & \text { in } D \times[0, T] \times \Omega, \\
\rho \frac{\partial u}{\partial t}-\nabla \cdot \epsilon(u)-\frac{\eta_{p}}{\lambda} \nabla \cdot\left(\mathbb{E}\left(\left(q^{D}+q^{S}\right) \otimes\left(q^{D}+q^{S}\right)\right)-I\right)+\nabla p=f & \text { in } D \times(0, T), \\
\nabla \cdot u=0 & \text { in } D \times(0, T), \\
u(., 0)=u_{0} & \text { in } D, \\
q(., 0, .)=0 & \text { in } D \times \Omega, \\
u=0 & \text { on } \partial D \times(0, T) .
\end{array}
$$

More precisely, given $q^{S} \in L^{\gamma}\left(\Omega ; h^{\mu}([0, T])\right)$ and $\left(f, u_{0}\right) \in Y$ small enough, there exists a unique $\left(u, q^{D}, p\right) \in$ $X \times h^{\mu}\left(W^{1, r} \cap L_{0}^{r}\right)$ solution to $(2.3)-(2.8)$ and the mapping

$$
\begin{aligned}
Y & \longrightarrow X \\
\left(f, u_{0}\right) & \longmapsto\left(u\left(f, u_{0}\right), q^{D}\left(f, u_{0}\right)\right)
\end{aligned}
$$


is analytic (see [19], Def. 4.3.1), therefore continuous. The space for the data $Y$ is a subset of $h^{\mu}\left(L^{r}\right) \times W^{2, r}$, which will be defined precisely later in this section. The above result is based on properties of the linearized problem: given $q_{0} \in L^{\gamma}(\Omega)$ satisfying $(1.8),\left(f_{1}, u_{0}\right) \in Y, f_{2} \in U$ and $w \in W$, find $\left(\tilde{u}, \tilde{q}^{D}, \tilde{p}\right) \in X \times h^{\mu}\left(W^{1, r} \cap L_{0}^{r}\right)$ such that

$$
\begin{array}{ll}
\rho \frac{\partial \tilde{u}}{\partial t}-2 \eta_{s} \nabla \cdot \epsilon(\tilde{u}) & \\
-\frac{\eta_{p}}{\lambda} \nabla \cdot\left(\mathbb{E}\left(\tilde{q}^{D} \otimes q^{S}+q^{S} \otimes \tilde{q}^{D}\right)+f_{2}\right)+\nabla \tilde{p}=f_{1} & \text { in } D \times(0, T), \\
\nabla \cdot \tilde{u}=0 & \text { in } D \times(0, T), \\
\frac{\partial \tilde{q}^{D}}{\partial t}+\frac{1}{2} \tilde{q}^{D}-(\nabla \tilde{u}) q^{S}=w & \text { in } D \times(0, T) \times \Omega, \\
\tilde{u}(\cdot, 0)=u_{0} & \text { in } D, \\
\tilde{q}^{D}(\cdot, 0)=0 & \text { in } \Omega, \\
\tilde{u}=0 & \text { on } \partial \Omega \times(0, T),
\end{array}
$$

where $q^{S} \in L^{\gamma}\left(\Omega ; h^{\mu}([0, T])\right)$ is defined by $(2.1)$,

$$
U=\left\{f_{2} \in h^{\mu}\left(W^{1, r}\right) ; \nabla \cdot f_{2}(0)=0\right\}
$$

and

$$
W=\left\{w \in L^{\gamma}\left(h^{\mu}\left(W^{1, r}\right)\right) ; w \text { adapted to }\left(\mathcal{F}_{t}\right)_{t \in[0, T]}\right\}
$$

are Banach spaces endowed with the norm of $h^{\mu}\left(W^{1, r}\right)$ and $L^{\gamma}\left(h^{\mu}\left(W^{1, r}\right)\right)$ respectively.

The space $Y$ is now defined. Let us introduce the Helmholtz-Weyl projector $P_{r}: L^{r} \rightarrow \mathcal{H}_{r}$, where $\mathcal{H}_{r}$ is the completion of the divergence free $C_{0}^{\infty}(D)$ vector fields with respect to the norm of $L^{r}$. The Stokes operator $A_{r}=-P_{r} \Delta$ with domain $\mathcal{D}_{A_{r}}=W^{2, r} \cap W_{0}^{1, r} \cap \mathcal{H}_{r}$ and range $\mathcal{H}_{r}$ will be necessary to characterize the space for the data $Y$. For this purpose, let

$$
E_{\mu, \infty}=\left(\mathcal{H}_{r}, \mathcal{D}_{A_{r}}\right)_{\mu, \infty}=\left\{x \in \mathcal{H}_{r} ; \sup _{t>0}\left\|t^{1-\mu} A_{r} e^{-t A_{r}} x\right\|_{L^{r}(D)}<+\infty\right\}
$$

be a Banach space endowed with the norm

$$
\|x\|_{E_{\mu, \infty}}=\|x\|_{L^{r}(D)}+\sup _{t>0}\left\|t^{1-\mu} A_{r} e^{-t A_{r}} x\right\|_{L^{r}(D)}
$$

We will consider the data $\left(f, u_{0}\right)$ belonging to $Y$ defined by

$$
Y=\left\{\left(f, u_{0}\right) \in h^{\mu}\left(L^{r}\right) \times \mathcal{D}_{A_{r}} \text { such that }-\eta_{s} A_{r} u_{0}+P_{r} f(0) \in{\overline{\mathcal{D}} A_{r}}^{E_{\mu, \infty}}\right\}
$$

provided with the norm $\|\cdot\|_{Y}$ defined by

$$
\left\|f, u_{0}\right\|_{Y}=\|f\|_{h^{\mu}\left(L^{r}\right)}+\left\|u_{0}\right\|_{W^{2, r}}+\left\|-\eta_{s} A_{r} u_{0}+P_{r} f(0)\right\|_{\overline{\mathcal{D}}_{A_{r}}}^{E_{\mu}, \infty} .
$$

Finally, it should be noted that since the differential equation (2.11) can be solved explicitly, then the solution $(\tilde{u}, \tilde{p}) \in h^{1+\mu}\left(L^{r}\right) \cap h^{\mu}\left(W^{2, r} \cap W_{0}^{1, r}\right) \times h^{\mu}\left(W^{1, r} \cap L_{0}^{r}\right)$ of (2.9)-(2.14) satisfies

$$
\rho \frac{\partial \tilde{u}}{\partial t}-\nabla \cdot\left(2 \eta_{s} \epsilon(\tilde{u})+2 k * \epsilon(\tilde{u})\right)+\nabla \tilde{p}=f_{1}+\frac{\eta_{p}}{\lambda} \nabla \cdot f_{2}+\nabla \cdot g, \quad \nabla \cdot \tilde{u}=0, \quad \tilde{u}(., 0)=u_{0}
$$


where $k \in \mathcal{C}^{\infty}([0, T])$ is defined for $t \in[0, T]$ by $k(t)=\frac{\eta_{p}}{\lambda} \mathrm{e}^{-\frac{t}{\lambda}}, g \in h_{0}^{\mu}\left(\mathcal{H}_{r}\right)$ is defined for $t \in[0, T]$ by

$$
g(t)=\frac{\eta_{p}}{\lambda} \int_{0}^{t} \mathrm{e}^{-\frac{t-s}{2 \lambda}} \mathbb{E}\left(w(s) \otimes q^{S}(t)+q^{S}(t) \otimes w(s)\right) \mathrm{d} s,
$$

and $k * \epsilon(\tilde{u})$ is the convolution in time of the kernel $k$ with $\epsilon(\tilde{u})$

$$
(k * \epsilon(\tilde{u}))(t)=\int_{0}^{t} k(t-s) \epsilon(\tilde{u}(s)) \mathrm{d} s .
$$

Moreover, there exists a constant $C>0$ independent of $f_{1}, f_{2}, u_{0}$ and $w$ such that

$$
\left\|\frac{\partial \tilde{u}}{\partial t}\right\|_{h^{\mu}\left(L^{r}\right)}+\left\|A_{r} \tilde{u}\right\|_{h^{\mu}\left(L^{r}\right)}+\|\tilde{p}\|_{h^{\mu}\left(W^{1, r}\right)} \leq C\left(\left\|f_{1}, u_{0}\right\|_{Y}+\left\|f_{2}\right\|_{h^{\mu}\left(W^{1, r}\right)}+\|w\|_{L^{\gamma}\left(h^{\mu}\left(W^{1, r}\right)\right)}\right) .
$$

In this paper, we will assume the results presented in this section still hold when $D$ is a convex polygon. Once again, the key point to prove these results when $D$ is a convex polygon is to prove that the negative Stokes operator $-A_{r}$ is still the generator of an analytic semi-group, see for instance [35].

\section{Existence AND A PRIORI ERROR ESTIMATES}

In order to prove that the solution of the nonlinear finite element discretization (1.9) exists and converges to that of (1.1)-(1.6), we introduce $X_{h} \subset X$ defined by

$$
X_{h}=L^{2}\left(V_{h}\right) \times L^{2}\left(L^{\infty}\left(R_{h}\right)\right),
$$

provided with the norm $\|\cdot\|_{X_{h}}$ defined for all $x_{h}=\left(u_{h}, q_{h}\right) \in X_{h}$ by

$$
\left\|x_{h}\right\|_{X_{h}}^{2}=2 \eta_{s} \int_{0}^{T}\left\|\epsilon\left(u_{h}(t)\right)\right\|_{L^{2}(D)}^{2} \mathrm{~d} t+\int_{\Omega} \sup _{t \in[0, T]}\left\|q_{h}(\omega, t)\right\|_{L^{2}(D)}^{2} \mathrm{~d} \mathcal{P}(\omega) .
$$

The splitting $q_{h}=q^{S}+q_{h}^{D}$ will also be used for the space discretization (remember $q^{S}$ does not depend on the space variable and satisfies $(2.1))$ where $q_{h}^{D} \in L^{2}\left(L^{\infty}\left(R_{h}\right)\right)$ satisfies

$$
\left(q_{h}^{D}(t), r_{h}\right)+\left(\int_{0}^{t}\left(\frac{1}{2 \lambda} q_{h}^{D}(k)-\left(\nabla u_{h}(k)\right)\left(q^{S}(k)+q_{h}^{D}(k)\right)\right) \mathrm{d} k, r_{h}\right)=0,
$$

for all $r_{h} \in R_{h}$, a.e. in $(0, T)$ and a.e. in $\Omega$.

It will be shown that there exists a unique $\left(u_{h}, q_{h}^{D}\right) \in X_{h}$ converging to $\left(u, q^{D}\right) \in X$ and thus a unique $\left(u_{h}, q_{h}\right)$ converging to $(u, q)$. For this purpose, the discrete problem corresponding to the unknowns $\left(u_{h}, q_{h}^{D}, p_{h}\right)$ will be written in the abstract framework of [20]. Using the splitting $q_{h}=q^{S}+q_{h}^{D}$, we rewrite the solution of (1.9) as the following fixed point problem. Given $y=\left(f, u_{0}\right) \in Y$, find $x_{h}=\left(u_{h}, q_{h}^{D}\right) \in X_{h}$ such that

$$
x_{h}=\mathrm{T}_{h}\left(y, S_{c}\left(x_{h}\right), S_{d}\left(x_{h}\right)\right)
$$

where

$$
\begin{aligned}
S_{c}: L^{2}\left(H^{1}\right) \times L^{2}\left(L^{\infty}\left(L^{2}\right)\right) & \longrightarrow L^{2}\left(L^{2}\right) \\
x_{h}=\left(u_{h}, q_{h}^{D}\right) & \longmapsto S_{c}\left(x_{h}\right)=\mathbb{E}\left(q_{h}^{D} \otimes q_{h}^{D}\right),
\end{aligned}
$$




$$
\begin{aligned}
S_{d}: L^{2}\left(H^{1}\right) \times L^{2}\left(L^{\infty}\left(L^{2}\right)\right) & \longrightarrow L^{2}\left(L^{2}\left(L^{2}\right)\right) \\
x_{h}=\left(u_{h}, q_{h}^{D}\right) & \longmapsto S_{d}\left(x_{h}\right)=\left(\nabla u_{h}\right) q_{h}^{D} .
\end{aligned}
$$

The linear operator $\mathrm{T}_{h}: Y \times L^{2}\left(L^{2}\right) \times L^{2}\left(L^{2}\left(L^{2}\right)\right) \longrightarrow X_{h}$ is defined as follow

$$
\left(f_{1}, u_{0}, f_{2}, w\right) \longmapsto \mathrm{T}_{h}\left(f_{1}, u_{0}, f_{2}, w\right)=\left(\tilde{u}_{h}, \tilde{q}_{h}^{D}\right) \in X_{h},
$$

where for almost all $t \in(0, T)$ and almost all $\omega \in \Omega$

$$
\left(\tilde{u}_{h}, \tilde{q}_{h}^{D}, \tilde{p}_{h}\right):(\omega, t) \longmapsto\left(\tilde{u}_{h}(t), \tilde{q}_{h}^{D}(\omega, t), \tilde{p}_{h}(t)\right) \in V_{h} \times R_{h} \times Q_{h}
$$

satisfies $\tilde{u}_{h}(0)=i_{h} u_{0}$ and

$$
\begin{array}{r}
\rho\left(\frac{\partial \tilde{u}_{h}}{\partial t}, v_{h}\right)+2 \eta_{s}\left(\epsilon\left(\tilde{u}_{h}\right), \epsilon\left(v_{h}\right)\right)-\left(\tilde{p}_{h}, \nabla \cdot v_{h}\right)+\frac{\eta_{p}}{\lambda}\left(\mathbb{E}\left(\tilde{q}_{h}^{D} \otimes q^{S}+q^{S} \otimes \tilde{q}_{h}^{D}\right)+f_{2}, \epsilon\left(v_{h}\right)\right) \\
-\left(f_{1}, v_{h}\right)+\left(\nabla \cdot \tilde{u}_{h}, s_{h}\right)+\sum_{K \in \mathcal{T}_{h}} \frac{\alpha h_{K}^{2}}{2 \eta_{p}}\left(\nabla \tilde{p}_{h}, \nabla s_{h}\right)_{K} \\
\left(\tilde{q}_{h}^{D}(t), r_{h}\right)+\left(\int_{0}^{t}\left(\frac{1}{2 \lambda} \tilde{q}_{h}^{D}(k)-\left(\nabla \tilde{u}_{h}(k)\right) q^{S}(k)-w\right) \mathrm{d} k, r_{h}\right)=0
\end{array}
$$

for all $\left(v_{h}, r_{h}, s_{h}\right) \in V_{h} \times R_{h} \times Q_{h}$, a.e. in $(0, T)$ and a.e. in $\Omega$.

It should be noticed that, given $y=\left(f, u_{0}\right) \in Y$ sufficiently small, the solution $x(y)=\left(u(y), q^{D}(y)\right) \in X$ of the continuous Hookean dumbbells problem (2.3)-(2.8) also satisfies a fixed point problem, namely

$$
x(y)=\mathrm{T}\left(y, S_{c}(x(y)), S_{d}(x(y))\right) .
$$

Here the operator $\mathrm{T}$ is defined by

$$
\begin{aligned}
& \mathrm{T}: Y \times U \times W \rightarrow X \\
& \quad\left(f_{1}, u_{0}, f_{2}, w\right) \rightarrow \mathrm{T}\left(f_{1}, u_{0}, f_{2}, w\right) \underset{\text { def. }}{=}\left(\tilde{u}, \tilde{q}^{D}\right),
\end{aligned}
$$

where $\left(\tilde{u}, \tilde{q}^{D}, \tilde{p}\right) \in X \times h^{\mu}\left(W^{1, r} \cap L_{0}^{2}\right)$ satisfy (2.9)-(2.14). Problem (3.7) is well defined since it has been proved that for $x=\left(u, q^{D}\right) \in X$ we have $S_{c}(x) \in h^{\mu}\left(W^{1, r}\right)$ and $S_{d}(x) \in W$ (see Rem. 3.6 in [11]). Moreover, since $q^{D}(0)=0$, it follows that $S_{c}(x)=\mathbb{E}\left(q^{D} \otimes q^{D}\right)$ vanishes at time $t=0$ and thus $S_{c}(x) \in U$ for $x \in X$.

The elongation vector $\tilde{q}_{h}^{D}$ can be eliminated from (3.6) and the next Lemma provides the equation satisfied by $\tilde{u}_{h}$. This equation is a discrete approximation of $(2.15)$.

Lemma 3.1. Let $\gamma \geq 2,0<\mu<1 / 2$ and $r>2$. Let $\left(f_{1}, u_{0}\right) \in Y, f_{2} \in L^{2}\left(L^{2}\right), w \in L^{2}\left(L^{2}\left(L^{2}\right)\right)$ and let $q^{S} \in L^{\gamma}\left(h^{\mu}\left(W^{1, r}\right)\right)$ be defined by (2.1). Then problem (3.6) admits a unique solution $\left(\tilde{u}_{h}, \tilde{q}_{h}^{D}\right) \in X_{h}$. Moreover, $\left(\tilde{u}_{h}, \tilde{p}_{h}\right)$ satisfies

$$
\begin{aligned}
\rho\left(\frac{\partial \tilde{u}_{h}}{\partial t}, v_{h}\right)+2 \eta_{s}\left(\epsilon\left(\tilde{u}_{h}\right), \epsilon\left(v_{h}\right)\right) & -\left(\tilde{p}_{h}, \nabla \cdot v_{h}\right)+2\left(k * i_{h} \epsilon\left(\tilde{u}_{h}\right), i_{h} \epsilon\left(v_{h}\right)\right) \\
& +\left(\nabla \cdot \tilde{u}_{h}, s_{h}\right)+\sum_{K \in \mathcal{T}_{h}} \frac{\alpha h_{K}^{2}}{2 \eta_{p}}\left(\nabla \tilde{p}_{h}, \nabla s_{h}\right)_{K}=\left(f_{1}, v_{h}\right)+\left(-\frac{\eta_{p}}{\lambda} f_{2}-i_{h} g, \epsilon\left(v_{h}\right)\right)
\end{aligned}
$$

where $k \in \mathcal{C}^{\infty}([0, T])$ is defined by $k(t)=\frac{\eta_{p}}{\lambda} e^{-t / \lambda}$ and where $g \in L^{\gamma}\left(h^{\mu}\left(W^{1, r}\right)\right)$ is defined by (2.16) and where $i_{h}$ is the $L^{2}(D)$ projection onto $R_{h} \otimes R_{h}$. 
Proof. In order to prove the existence (and uniqueness) of a solution $\left(\tilde{u}_{h}, \tilde{q}_{h}^{D}\right)$, we will write (3.6) using a basis of $R_{h}$. Hence, let us introduce $\varphi_{i}^{n}, n=1,2, i=1, \ldots, P$ an orthonormal basis of $R_{h}$ where $P$ is the number of nodes of the mesh. Let $\tilde{q}_{h, i}^{D, n}$ be the components of $q_{h}^{D}$ and $\tilde{u}_{h, i}^{n}$ be those of $u_{h}$ with respect to the given basis $\varphi_{i}^{n}$. We have

$$
\begin{aligned}
\tilde{q}_{h}^{D}(\omega, t, x) & =\sum_{n=1}^{2} \sum_{i=1}^{P} q_{h, i}^{D, n}(\omega, t) \varphi_{i}^{n}(x), \\
\tilde{u}_{h}(t, x) & =\sum_{n=1}^{2} \sum_{i=1}^{P} u_{h, i}^{n}(t) \varphi_{i}^{n}(x) .
\end{aligned}
$$

Choosing $v_{h}=0, s_{h}=0, r_{h}=\varphi_{i}^{n}$ in (3.6) we have

$$
\tilde{q}_{h, i}^{D, n}(t)=\int_{0}^{t} \mathrm{e}^{-\frac{t-s}{2 \lambda}}\left(\sum_{k=1}^{2} \frac{\partial \tilde{u}_{h}^{n}}{\partial x_{k}}(s) q^{S, k}(s)+w^{n}, \varphi_{i}^{n}\right) \mathrm{d} s, \quad n=1,2, i=1, \ldots, P,
$$

a.e. in $(0, T)$, a.e. in $\Omega$ and with $w=\left(w^{1}, w^{2}\right)^{T}$. The definition of the $L^{2}(D)$ projection $i_{h}$ implies for $t \in[0, T]$

$$
\tilde{q}_{h}^{D}(t)=\int_{0}^{t} \mathrm{e}^{-\frac{t-s}{2 \lambda}}\left(i_{h}\left(\nabla \tilde{u}_{h}\right) q^{S}+i_{h} w\right) \mathrm{d} s
$$

a.e. in $\Omega$. Going back to (3.6) we find that $\left(\tilde{u}_{h}, \tilde{p}_{h}\right)$ satisfies

$$
\begin{aligned}
\rho\left(\frac{\partial \tilde{u}_{h}}{\partial t}, v_{h}\right)+2 \eta_{s}\left(\epsilon\left(\tilde{u}_{h}\right), \epsilon\left(v_{h}\right)\right)- & \left(\tilde{p}_{h}, \nabla \cdot v_{h}\right)+2\left(k * i_{h} \epsilon\left(u_{h}\right), \epsilon\left(v_{h}\right)\right) \\
& +\left(\nabla \cdot \tilde{u}_{h}, s_{h}\right)+\sum_{K \in \mathcal{T}_{h}} \frac{\alpha h_{K}^{2}}{2 \eta_{p}}\left(\nabla \tilde{p}_{h}, \nabla s_{h}\right)_{K}=\left(f_{1}, v_{h}\right)+\left(-\frac{\eta_{p}}{\lambda} f_{2}-i_{h} g, \epsilon\left(v_{h}\right)\right) .
\end{aligned}
$$

Using the property of the $L^{2}$-projection

$$
\left(i_{h} \epsilon\left(\tilde{u}_{h}\right), \epsilon\left(v_{h}\right)-i_{h} \epsilon\left(v_{h}\right)\right)=0 \quad \forall v_{h} \in V_{h},
$$

we obtain (3.9). Thus, problem (3.6) is equivalent to (3.9) and (3.10). Existence (and uniqueness) of $\tilde{u}_{h} \in$ $\mathcal{C}^{1}\left([0, T] ; V_{h}\right)$ satisfying (3.9) is ensured by a standard argument on Stokes system (see for instance [60]) and a contraction mapping theorem (see for instance [45] or App. A in [10]). Finally, since $q^{S} \in L^{\gamma}\left(h^{\mu}\left(W^{1, r}\right)\right)$, equation (3.10) ensures the existence (and uniqueness) of $\tilde{q}_{h}^{D} \in L^{\gamma}\left(\mathcal{C}^{1}\left(R_{h}\right)\right)$ thus in $L^{2}\left(L^{\infty}\left(R_{h}\right)\right)$.

Remark 3.2. We proved in the previous Lemma that $\tilde{q}_{h}^{D}$ belongs to $L^{\gamma}\left(\mathcal{C}^{1}\left(R_{h}\right)\right)$, thus (3.1) can be rewritten

$$
\left(\frac{\partial q_{h}^{D}}{\partial t}, r_{h}\right)+\left(\frac{1}{2 \lambda} q_{h}^{D}, r_{h}\right)-\left(\left(\nabla u_{h}\right)\left(q^{S}+q_{h}^{D}\right), r_{h}\right)=0 \quad \forall r_{h} \in R_{h}, \quad \text { a.e. in } \Omega .
$$

We have the following stability and convergence result.

Lemma 3.3. The operator $\mathrm{T}_{h}$ is well defined and uniformly bounded with respect to $h$ : there exists $C_{1}>0$ such that for all $h>0$ and for $\left(f_{1}, u_{0}\right) \in Y, f_{2} \in L^{2}\left(L^{2}\right), w \in L^{2}\left(L^{2}\left(L^{2}\right)\right)$ we have

$$
\left\|\mathrm{T}_{h}\left(f_{1}, u_{0}, f_{2}, w\right)\right\|_{X_{h}} \leq C_{1}\left(\left\|f_{1}, u_{0}\right\|_{Y}+\left\|f_{2}\right\|_{L^{2}\left(L^{2}\right)}+\|w\|_{L^{2}\left(L^{2}\left(L^{2}\right)\right)}\right) .
$$


Moreover, there exists $C_{2}>0$ such that for all $h>0$ and for all $\left(f_{1}, u_{0}, f_{2}, w\right) \in Y \times U \times W$, we have

$$
\left\|\left(\mathrm{T}-\mathrm{T}_{h}\right)\left(f_{1}, u_{0}, f_{2}, w\right)\right\|_{X_{h}} \leq C_{2} h\left(\left\|f_{1}, u_{0}\right\|_{Y}+\left\|f_{2}\right\|_{U}+\|w\|_{W}\right) .
$$

Proof. Let us use the notation $\left(\tilde{u}_{h}, \tilde{q}_{h}^{D}\right)=\mathrm{T}_{h}\left(f_{1}, u_{0}, f_{2}, w\right)$, where $\left(\tilde{u}_{h}, \tilde{q}_{h}^{D}\right) \in X_{h}$ satisfies (3.9). From Lemma 1 in [66], we have

$$
\int_{0}^{T} \int_{0}^{t} \mathrm{e}^{-\frac{t-s}{\lambda}}\left(i_{h} \epsilon\left(\tilde{u}_{h}(s)\right), i_{h} \epsilon\left(\tilde{u}_{h}(t)\right)\right) \mathrm{d} s \mathrm{~d} t \geq 0 .
$$

Therefore, choosing $v_{h}=u_{h}(t)$ in (3.9), there exists a constant $C$ independent of $f_{1}, f_{2}, g$ such that

$$
\left\|\tilde{u}_{h}\right\|_{L^{2}\left(H^{1}\right)} \leq C\left(\left\|f_{1}, u_{0}\right\|_{Y}+\left\|f_{2}\right\|_{L^{2}\left(L^{2}\right)}+\left\|i_{h} g\right\|_{L^{2}\left(L^{2}\right)}\right)
$$

where $g \in h_{0}^{\mu}\left(W^{1, r}\right)$ is defined by (2.16). Moreover since $i_{h}$ is bounded in $L^{2}(D)$, using the continuous embedding $h^{\mu}([0, T]) \subset_{>} L^{\infty}([0, T])$ and Cauchy-Schwarz inequality, we have

$$
\left\|i_{h} g\right\|_{L^{2}\left(L^{2}\right)} \leq C\left\|q^{S}\right\|_{L^{2}\left(h^{\mu}\right)}\|w\|_{L^{2}\left(L^{2}\left(L^{2}\right)\right)}
$$

where $C$ is a constant independent of $h, w, q^{S}$ and $g$.

On the other hand from (3.10) we have

$$
\left\|\tilde{q}_{h}^{D}\right\|_{L^{2}\left(L^{\infty}\left(L^{2}\right)\right)} \leq C\left(\left\|\tilde{u}_{h}\right\|_{L^{2}\left(H^{1}\right)}+\|w\|_{L^{2}\left(L^{2}\left(L^{2}\right)\right)}\right)
$$

where $C$ is a constant independent of $h, f_{1}, u_{0}, f_{2}$ and $w$. Thus (3.16) in (3.15) and (3.17) leads to (3.12). The proof of (3.13) is provided in Appendix A.

Our goal is now to prove that (1.9) has a unique solution $\left(u_{h}, q_{h}\right)$ converging to that of $(2.3)-(2.8)$. Since $q^{S}$ does not depend on $x \in D$, it suffices to show that (3.2) has a unique solution $\left(u_{h}, q_{h}^{D}\right)$ converging to $\left(u, q^{D}\right)$ solution of (1.1)-(1.6). For this purpose, we use, as in [10,59], an abstract framework and write (1.9) as the following problem : given $y=\left(f, u_{0}\right) \in Y$, find $x_{h}=\left(u_{h}, q_{h}^{D}\right) \in X_{h}$ such that

$$
F_{h}\left(y, x_{h}\right)=0
$$

where $F_{h}: Y \times X_{h} \rightarrow X_{h}$ is defined by

$$
F_{h}\left(y, x_{h}\right)=x_{h}-\mathrm{T}_{h}\left(y, S_{c}\left(x_{h}\right), S_{d}\left(x_{h}\right)\right)
$$

In order to prove existence and convergence, we use Theorem 2.1 of [20]. The mapping $F_{h}: Y \times X_{h} \rightarrow X_{h}$ is $\mathcal{C}^{1}$. We first prove that the scheme is consistent and that $D_{x} F_{h}$ is locally Lipschitz.

Lemma 3.4. Let $y=\left(f, u_{0}\right) \in Y$ be sufficiently small, let $x(y)=\left(u(y), q^{D}(y)\right) \in X$ be the solution of (2.3)-(2.8). Then, there exists a constant $C_{1}$ such that for all $0<h<1$, for all $y \in Y$ we have

$$
\left\|F_{h}\left(y, i_{h} x(y)\right)\right\|_{X_{h}} \leq C_{1} h\left(\|y\|_{Y}+\|x(y)\|_{X}+\|x(y)\|_{X}^{2}\right)
$$

Moreover, there exists a constant $C_{2}$ such that for all $h>0$, for all $y \in Y$, for all $z \in X_{h}$ we have

$$
\left\|D_{x} F_{h}\left(y, i_{h} x(y)\right)-D_{x} F_{h}(y, z)\right\|_{\mathcal{L}\left(X_{h}\right)} \leq \frac{C_{2}}{h}\left\|i_{h} x(y)-z\right\|_{X_{h}}
$$


Proof. From the definition of $F_{h}$ we have

$$
\begin{aligned}
F_{h}\left(y, i_{h} x\right) & =i_{h} x-\mathrm{T}_{h}\left(y, S_{c}\left(i_{h} x\right), S_{d}\left(i_{h} x\right)\right) \\
& =\left(i_{h} x-x\right)+\mathrm{T}_{h}\left(0,0, S_{c}(x)-S_{c}\left(i_{h} x\right), S_{d}(x)-S_{d}\left(i_{h} x\right)\right)+\left(\mathrm{T}-\mathrm{T}_{h}\right)\left(y, S_{c}(x), S_{d}(x)\right)
\end{aligned}
$$

so that,

$$
\begin{aligned}
\frac{1}{3}\left\|F_{h}\left(y, i_{h} x\right)\right\|_{X_{h}}^{2} \leq & \left\|i_{h} x-x\right\|_{X_{h}}^{2}+\left\|\mathrm{T}_{h}\left(0,0, S_{c}(x)-S_{c}\left(i_{h} x\right), S_{d}(x)-S_{d}\left(i_{h} x\right)\right)\right\|_{X_{h}}^{2} \\
& +\left\|\left(\mathbf{T}-\mathrm{T}_{h}\right)\left(y, S_{c}(x), S_{d}(x)\right)\right\|_{X_{h}}^{2} .
\end{aligned}
$$

Using standard interpolation results for the first term of the right hand side, Lemma 3.3 for the second and third terms, it follows that

$$
\begin{aligned}
\left\|F_{h}\left(y, i_{h} x\right)\right\|_{X_{h}}^{2} \leq C\left(h^{2}\|x\|_{X}^{2}+\| S_{c}(x)-\right. & S_{c}\left(i_{h} x\right)\left\|_{L^{2}\left(L^{2}\right)}^{2}+\right\| S_{d}(x)-S_{d}\left(i_{h} x\right) \|_{L^{2}\left(L^{2}\left(L^{2}\right)\right)}^{2} \\
& \left.+h^{2}\|y\|_{Y}^{2}+h^{2}\left\|S_{c}(x)\right\|_{h^{\mu}\left(W^{1, r}\right)}+h^{2}\left\|S_{d}(x)\right\|_{L^{2}\left(h^{\mu}\left(W^{1, r}\right)\right)}^{2}\right)
\end{aligned}
$$

$C$ being independent of $h$ and $y$. Proceeding as in Corollary 3.5 in [11], we have

$$
\left\|S_{c}(x)\right\|_{h^{\mu}\left(W^{1, r}\right)}+\left\|S_{d}(x)\right\|_{L^{2}\left(h^{\mu}\left(W^{1, r}\right)\right)}=\left\|\frac{\lambda}{\eta_{p}} \mathbb{E}\left(q^{D} \otimes q^{D}\right)\right\|_{h^{\mu}\left(W^{1, r}\right)}+\left\|(\nabla u) q^{D}\right\|_{L^{2}\left(h^{\mu}\left(W^{1, r}\right)\right)} \leq C\|x\|_{X}^{2},
$$

$C$ being independent of $h$ and $y$. On the other hand, we also have

$$
\begin{aligned}
S_{d}(x)-S_{d}\left(i_{h} x\right) & =(\nabla u) q^{D}-\left(\nabla i_{h} u\right) i_{h} q^{D} \\
& =\left(\nabla\left(u-i_{h} u\right)\right) q^{D}+\left(\nabla i_{h} u\right)\left(q^{D}-i_{h} q^{D}\right)
\end{aligned}
$$

so that, using Cauchy-Schwarz inequality

$$
\left\|S_{d}(x)-S_{d}\left(i_{h} x\right)\right\|_{L^{2}\left(L^{2}\left(L^{2}\right)\right)} \leq C\left\|x-i_{h} x\right\|_{X_{h}}\left(\left\|q^{D}\right\|_{L^{2}\left(L^{\infty}\left(L^{\infty}\right)\right)}+\left\|\nabla i_{h} u\right\|_{L^{2}\left(L^{\infty}\right)}\right)
$$

$C$ being independent of $h$ and $y$. Standard interpolation results lead to

$$
\left\|x-i_{h} x\right\|_{X_{h}} \leq C_{1} h\|x\|_{X}
$$

and

$$
\left\|\nabla i_{h} u\right\|_{L^{2}\left(L^{\infty}\right)} \leq\|\nabla u\|_{L^{2}\left(L^{\infty}\right)}+\left\|\nabla\left(u-i_{h} u\right)\right\|_{L^{2}\left(L^{\infty}\right)} \leq C_{2}\|u\|_{h^{\mu}\left(W^{2, r}\right)},
$$

for $0<h<1$ and where $C_{1}, C_{2}$ are constants independent of $h$ and $x$. Thus we obtain

$$
\left\|S_{d}(x)-S_{d}\left(i_{h} x\right)\right\|_{L^{2}\left(L^{2}\left(L^{2}\right)\right)} \leq C h\|x\|_{X},
$$

$C$ being independent of $h$ and $y$. Similarly, we obtain

$$
\left\|S_{c}(x)-S_{c}\left(i_{h} x\right)\right\|_{L^{2}\left(L^{2}\right)} \leq C h\|x\|_{X} .
$$

Finally, (3.22), (3.23) and (3.24) in (3.21) yields (3.19). 
Let us now prove $(3.20)$. Let $z=(v, r) \in X_{h}$, let $\tilde{z}=(\tilde{v}, \tilde{r}) \in X_{h}$, we have

$$
\left(D_{x} F_{h}\left(y, i_{h} x\right)-D_{x} F_{h}(y, z)\right) \tilde{z}=-\mathrm{T}_{h}\left(0,0,\left(D S_{c}\left(i_{h} x\right)-D S_{c}(z)\right) \tilde{z},\left(D S_{d}\left(i_{h} x\right)-D S_{d}(z)\right) \tilde{z}\right) .
$$

Using Lemma 3.3 we obtain

$$
\begin{aligned}
& \left\|\left(D_{x} F_{h}\left(y, i_{h} x\right)-D_{x} F_{h}(y, z)\right) \tilde{z}\right\|_{X_{h}} \\
& \quad \leq C\left(\left\|\left(D S_{c}\left(i_{h} x\right)-D S_{c}(z)\right) \tilde{z}\right\|_{L^{2}\left(L^{2}\right)}+\left\|\left(D S_{d}\left(i_{h} x\right)-D S_{d}(z)\right) \tilde{z}\right\|_{L^{2}\left(L^{2}\left(L^{2}\right)\right)}\right) \\
& \quad
\end{aligned}
$$

$C$ being independent of $h$ and $y$. Using Cauchy-Schwarz inequality, there exists a constant $C$ independent of $h$ and $y$ such that

$\left\|\left(D S_{d}\left(i_{h} x\right)-D S_{d}(z)\right) \tilde{z}\right\|_{L^{2}\left(L^{2}\left(L^{2}\right)\right)} \leq C\left(\left\|\nabla\left(i_{h} u-v\right)\right\|_{L^{2}\left(L^{\infty}\right)}\|\tilde{r}\|_{L^{2}\left(L^{\infty}\left(L^{2}\right)\right)}+\|\nabla \tilde{v}\|_{L^{2}\left(L^{\infty}\right)}\left\|i_{h} q^{D}-r\right\|_{L^{2}\left(L^{\infty}\left(L^{2}\right)\right)}\right)$.

A classical inverse inequality yields

$\left\|\left(D S_{d}\left(i_{h} x\right)-D S_{d}(z)\right) \tilde{z}\right\|_{L^{2}\left(L^{2}\left(L^{2}\right)\right)} \leq \frac{\tilde{C}}{h}\left(\left\|\nabla\left(i_{h} u-v\right)\right\|_{L^{2}\left(L^{2}\right)}\|\tilde{r}\|_{L^{2}\left(L^{\infty}\left(L^{2}\right)\right)}+\|\nabla \tilde{v}\|_{L^{2}\left(L^{2}\right)}\left\|i_{h} q^{D}-r\right\|_{L^{2}\left(L^{\infty}\left(L^{2}\right)\right)}\right)$

$\tilde{C}$ being independent of $h$ and $y$, so that we finally have

$$
\left\|\left(D S_{d}\left(i_{h} x\right)-D S_{d}(z)\right) \tilde{z}\right\|_{L^{2}\left(L^{2}\left(L^{2}\right)\right)} \leq \frac{\tilde{C}}{h}\left\|i_{h} x-z\right\|_{X_{h}}\|\tilde{z}\|_{X_{h}} .
$$

Similarly, we obtain

$$
\begin{aligned}
\left\|\left(D S_{c}\left(i_{h} x\right)-D S_{c}(z)\right) \tilde{z}\right\|_{L^{2}\left(0, T ; L^{2}(D)\right)} & \leq C\left\|i_{h} q-r\right\|_{L^{2}\left(L^{\infty}\left(L^{\infty}\right)\right)}\|\tilde{r}\|_{L^{2}\left(L^{\infty}\left(L^{2}\right)\right)} \\
& \leq \frac{\tilde{C}}{h}\left\|i_{h} x-z\right\|_{X_{h}}\|\tilde{z}\|_{X_{h}} .
\end{aligned}
$$

Inequalities (3.26) and (3.27) in (3.25) yields (3.20).

Before proving existence of a solution to (3.18) we still need to check that $D_{x} F_{h}\left(y, i_{h} x\right)$ is invertible.

Lemma 3.5. Let $y=\left(f, u_{0}\right) \in Y$ be sufficiently small, let $x(y)=\left(u(y), q^{D}(y)\right) \in X$ be the solution of (2.3)-(2.8). Then, for $y$ sufficiently small, for all $h \leq 1$ we have

$$
\left\|D_{x} F_{h}\left(y, i_{h} x(y)\right)^{-1}\right\|_{\mathcal{L}\left(X_{h}\right)} \leq 2
$$

Proof. By definition of $F_{h}$, we have

$$
D_{x} F_{h}\left(y, i_{h} x\right)=I-\mathrm{T}_{h}\left(0,0, D S_{c}\left(i_{h} x\right), D S_{d}\left(i_{h} x\right)\right),
$$

so that we can write

$$
D_{x} F_{h}\left(y, i_{h} x\right)=I-G_{h} \quad \text { with } \quad G_{h}=\mathrm{T}_{h}\left(0,0, D S_{c}\left(i_{h} x\right), D S\left(i_{h} x\right)\right) .
$$

If we prove that $\left\|G_{h}\right\|_{\mathcal{L}\left(X_{h}\right)} \leq 1 / 2$ for $y$ sufficiently small, then $D_{x} F_{h}\left(y, i_{h} x\right)$ is invertible and

$$
\left\|D_{x} F_{h}\left(y, i_{h} x\right)^{-1}\right\|_{\mathcal{L}\left(X_{h}\right)} \leq 2
$$


Let $z=(v, \tau) \in X_{h}$. Using Lemma 3.3 we have

$$
\left\|G_{h}(z)\right\|_{X_{h}} \leq C_{1}\left(\left\|D S_{c}\left(i_{h} x\right) z\right\|_{L^{2}\left(L^{2}\right)}+\left\|D S_{d}\left(i_{h} x\right) z\right\|_{L^{2}\left(L^{2}\left(L^{2}\right)\right)}\right)
$$

$C_{1}$ being independent of $y$ and $h$. Proceeding as in the proof of Lemma 3.4, we have

$$
\left\|D S_{d}\left(i_{h} x\right) z\right\|_{L^{2}\left(L^{2}\left(L^{2}\right)\right)} \leq C_{2}\left(\|\nabla u\|_{L^{2}\left(W^{1, r}\right)}\|\tau\|_{L^{2}\left(L^{\infty}\left(L^{2}\right)\right)}+\|\nabla v\|_{L^{2}\left(L^{2}\right)}\left\|q^{D}\right\|_{L^{2}\left(L^{\infty}\left(W^{1, r}\right)\right)}\right),
$$

$C_{2}$ being independent of $y, h$ and $z$. Hence,

$$
\left\|G_{h}(z)\right\|_{X_{h}} \leq C_{3}\|x\|_{X}\|z\|_{X_{h}}
$$

where $C_{3}$ is independent of $y, h$ and $z$. From Lemma 3.10 in [11], the mapping $y \rightarrow x(y)$ is continuous, thus if $\|y\|_{Y}$ is sufficiently small we have $\|x\|_{X} \leq 1 /\left(2 C_{3}\right)$ so that

$$
\left\|G_{h}(z)\right\|_{X_{h}} \leq \frac{1}{2}\|z\|_{X_{h}}
$$

We can now prove existence of a solution to the finite element scheme (1.9) and convergence to the solution of $(1.1)-(1.6)$.

Theorem 3.6. Let $y=\left(f, u_{0}\right) \in Y$ be sufficiently small, let $x(y)=\left(u(y), q^{D}(y)\right) \in X$ be the solution of (2.3)-(2.8). Then, there exists $\zeta>0$ such that for $y$ and $h$ sufficiently small, there exists a unique $x_{h}(y)=\left(u_{h}(y), q_{h}^{D}(y)\right)$ in the ball of $X_{h}$ centered at $i_{h} x(y)$ with radius $\zeta h$, satisfying

$$
F_{h}\left(y, x_{h}(y)\right)=0
$$

Moreover, the mapping $y \rightarrow x_{h}(y)$ is continuous and there exists $C>0$ independent of $h$ and $y$ such that the following a priori error estimate holds

$$
\left\|x(y)-x_{h}(y)\right\|_{X_{h}} \leq C h .
$$

In order to prove the above theorem, we will use the following abstract result.

Lemma 3.7 (Thm. 2.1 of [20]). Let $Y$ and $Z$ be two real Banach spaces with norms $\|\cdot\|_{Y}$ and $\|\cdot\|_{Z}$ respectively. Let $G: Y \rightarrow Z$ be a $\mathcal{C}^{1}$ mapping and $v \in Y$ be such that $D G(v) \in \mathcal{L}(Y ; Z)$ is an isomorphism. We introduce the notations

$$
\begin{gathered}
\epsilon=\|G(v)\|_{Z}, \\
\gamma=\left\|D G(v)^{-1}\right\|_{\mathcal{L}(Y ; Z)}, \\
L(\alpha)=\sup _{x \in \bar{B}(v, \alpha)}\|D G(v)-D G(x)\|_{\mathcal{L}(Y ; Z)},
\end{gathered}
$$

with $\bar{B}(v, \alpha)=\left\{y \in Y ;\|v-y\|_{Y} \leq \alpha\right\}$, and we are interested in finding $u \in Y$ such that

$$
G(u)=0
$$

We assume that $2 \gamma L(2 \gamma \epsilon) \leq 1$. Then Problem (3.29) has a unique solution $u$ in the ball $\bar{B}(v, 2 \gamma \epsilon)$ and, for all $x \in \bar{B}(v, 2 \gamma \epsilon)$, we have

$$
\|x-u\|_{Y} \leq 2 \gamma\|G(x)\|_{Z}
$$


Proof of Theorem 3.6. We apply Lemma 3.7 with $Y=X_{h}, Z=X_{h}, G=F_{h}$ and $v=i_{h} x(y)$. According to Lemma 3.4 there exists a constant $C_{1}$ independent of $y$ and $h$ such that

$$
\epsilon=\left\|F_{h}\left(y, i_{h} x(y)\right)\right\|_{X_{h}} \leq C_{1} h\left(\|y\|_{Y}+\|x(y)\|_{X}+\|x(y)\|_{X}^{2}\right) .
$$

According to Lemma 3.5, for $\|y\|_{Y}$ sufficiently small

$$
\gamma=\left\|D_{x} F_{h}\left(y, i_{h} x(y)\right)\right\|_{\mathcal{L}\left(X_{h}\right)} \leq 2
$$

According to Lemma 3.4, there is a constant $C_{2}$ independent of $y$ and $h$ such that

$$
L(\alpha)=\sup _{x \in \bar{B}\left(i_{h} x(y), \alpha\right)}\left\|D F_{h}\left(i_{h} x(y)\right)-D F_{h}(x)\right\|_{\mathcal{L}\left(X_{h}\right)} \leq \frac{C_{2}}{h} \alpha .
$$

Hence, we have

$$
\begin{aligned}
2 \gamma L(2 \gamma \epsilon) & \leq 2.2 \frac{C_{2}}{h}\left(2.2 C_{1} h\left(\|y\|_{Y}+\|x(y)\|_{X}+\|x(y)\|_{X}^{2}\right)\right) \\
& =16 C_{1} C_{2}\left(\|y\|_{Y}+\|x(y)\|_{X}+\|x(y)\|_{X}^{2}\right) .
\end{aligned}
$$

Using the continuity of the mapping $y \rightarrow x(y)$ it follows that, for sufficiently small $y \in Y$

$$
\|y\|_{Y}+\|x(y)\|_{X}+\|x(y)\|_{X}^{2}<\frac{1}{16 C_{1} C_{2}},
$$

then $2 \gamma L(2 \gamma \epsilon)<1$ and Lemma 3.7 applies. There exists a unique $x_{h}(y)$ in the ball $\bar{B}\left(i_{h} x(y), 2 \gamma \epsilon\right)$ such that

$$
F_{h}\left(y, x_{h}(y)\right)=0
$$

and we have

$$
\begin{aligned}
\left\|i_{h} x(y)-x_{h}(y)\right\|_{X_{h}} \leq 2 \gamma \epsilon & \leq 4 C_{1} h\left(\|y\|_{Y}+\|x(y)\|_{X}+\|x(y)\|_{X}^{2}\right) \\
& \leq \frac{1}{4 C_{2}} h .
\end{aligned}
$$

It suffices to use the triangle inequality

$$
\left\|x(y)-x_{h}(y)\right\|_{X_{h}} \leq\left\|x(y)-i_{h} x(y)\right\|_{X_{h}}+\left\|i_{h} x(y)-x_{h}(y)\right\|_{X_{h}},
$$

and standard interpolation results to obtain (3.28). The fact that the mapping $y \rightarrow x_{h}(y)$ is continuous is a direct consequence of the implicit function theorem.

Corollary 3.8. Under the assumptions of Theorem 3.6, there exists a constant $C$ independent of $h$ and $y$ such that

$$
\sup _{t \in[0, T]} \int_{D}\left|\mathbb{E}\left(q^{D}(t) \otimes q^{D}(t)-q_{h}^{D}(t) \otimes q_{h}^{D}(t)\right)\right|^{2} \leq C\left\|q^{D}-q_{h}^{D}\right\|_{L^{2}\left(L^{\infty}\left(L^{2}\right)\right)}^{2} .
$$

Moreover, there exists a constant $C$ independent of $y$ and $h$ such that

$$
\sup _{t \in[0, T]} \int_{D}\left|\mathbb{E}\left(q^{D}(t) \otimes q^{D}(t)-q_{h}^{D}(t) \otimes q_{h}^{D}(t)\right)\right|^{2} \leq C h^{2} .
$$


Proof. Let us use the notation

$$
q^{D}=\left(\begin{array}{c}
q^{D, 1} \\
q^{D, 2}
\end{array}\right), \quad q_{h}^{D}=\left(\begin{array}{c}
q_{h}^{D, 1} \\
q_{h}^{D, 2}
\end{array}\right) .
$$

It follows, using Cauchy-Schwarz inequalities that

$$
\begin{aligned}
\sup _{t \in[0, T]} \int_{D}\left|\mathbb{E}\left(q^{D}(t) \otimes q^{D}(t)-q_{h}(t) \otimes q_{h}(t)\right)\right|^{2} & =\sup _{t \in[0, T]} \int_{D} \sum_{i, j=1}^{2} \mathbb{E}\left(q^{D, i}(t) q^{D, j}(t)-q_{h}^{D, i}(t) q_{h}^{D, j}(t)\right)^{2} \\
& \leq \sup _{t \in[0, T]} \int_{D} \sum_{i=1}^{2} \mathbb{E}\left(\left(q^{D, i}(t)-q_{h}^{D, i}(t)\right)^{2}\right) \sum_{i=1}^{2} \mathbb{E}\left(\left(q^{D, i}(t)+q_{h}^{D, i}(t)\right)^{2}\right) .
\end{aligned}
$$

Fubini's Theorem and an Hölder's inequality lead to

$$
\sup _{t \in[0, T]} \int_{D}\left|\mathbb{E}\left(q^{D}(t) \otimes q^{D}(t)-q_{h}^{D}(t) \otimes q_{h}^{D}(t)\right)\right|^{2} \leq\left\|q^{D}-q_{h}^{D}\right\|_{L^{2}\left(\Omega ; L^{\infty}\left(0, T ; L^{2}(D)\right)\right)}^{2}\left\|q^{D}+q_{h}^{D}\right\|_{L^{2}\left(\Omega ; L^{\infty}\left(0, T ; L^{\infty}(D)\right)\right)}^{2} .
$$

It remains to prove that the term $\left\|q^{D}+q_{h}^{D}\right\|_{L^{2}\left(\Omega ; L^{\infty}\left(0, T ; L^{\infty}(D)\right)\right)}^{2}$ is uniformly bounded with respect to $h$. Since $i_{h}$ is bounded from $L^{\infty}(D)$ to $W^{1, r}(D)$ we have

$$
\left\|q^{D}+q_{h}^{D}\right\|_{L^{2}\left(L^{\infty}\left(L^{\infty}\right)\right)} \leq\left\|q_{h}^{D}-i_{h} q^{D}\right\|_{L^{2}\left(L^{\infty}\left(L^{\infty}\right)\right)}+C\left\|q^{D}\right\|_{L^{2}\left(L^{\infty}\left(W^{1, r}\right)\right)},
$$

where $C$ is independent of $h$ and $y$. Using an inverse estimate we have

$$
\left\|q_{h}^{D}-i_{h} q^{D}\right\|_{L^{2}\left(L^{\infty}\left(L^{\infty}\right)\right)} \leq \frac{C}{h}\left\|q_{h}^{D}-i_{h} q^{D}\right\|_{L^{2}\left(L^{\infty}\left(L^{2}\right)\right)},
$$

where $C$ is independent of $h$. From (3.31) $\left\|q_{h}^{D}-i_{h} q^{D}\right\|_{L^{2}\left(L^{\infty}\left(L^{2}\right)\right)} \leq C h$ so that we obtain

$$
\left\|q^{D}+q_{h}^{D}\right\|_{L^{2}\left(L^{\infty}\left(L^{\infty}\right)\right)} \leq C,
$$

with $C$ a constant independent of $h$, which yields to the first estimate of the Corollary. The second estimate is a consequence of Theorem 3.6.

\section{A POSTERIORI ERROR ESTIMATES}

Let us consider again the operator $\mathrm{T}_{h}: Y \times L^{2}\left(L^{2}\right) \times L^{2}\left(L^{2}\left(L^{2}\right)\right) \rightarrow X_{h}$ defined by (3.5). A residual based error estimator for $\mathrm{T}_{h}$ is now introduced using the notations of [4]. For any triangle $K$ of the triangulation $\mathcal{T}_{h}$, let $E_{K}$ be the set of its three edges. For each interior edge $l$ of $\mathcal{T}_{h}$, let us choose an arbitrary normal direction $n$ and let $[.]_{l}$ denotes the jump of the inside quantity across edge $l$. For each edge $l$ of $\mathcal{T}_{h}$ lying on the boundary $\partial D$, we set $[.]_{l}=0$. The local error estimators corresponding to (3.6) are then defined by

$$
\begin{aligned}
\mu_{K}^{2}\left(f_{1}, u_{0}, f_{2}, w\right)=\int_{0}^{T} & \left\{h_{K}^{2}\left\|\rho \frac{\partial \tilde{u}_{h}}{\partial t}-2 \eta_{s} \nabla \cdot \epsilon\left(\tilde{u}_{h}\right)+\nabla \tilde{p}_{h}-2 k * \nabla \cdot \epsilon\left(\tilde{u}_{h}\right)-f_{1}-\frac{\eta_{p}}{\lambda} \nabla \cdot f_{2}-\nabla \cdot g\right\|_{L^{2}(K)}^{2}\right. \\
& +\left\|\nabla \cdot \tilde{u}_{h}\right\|_{L^{2}(K)}^{2}+\frac{1}{2} \sum_{l \in E_{K}}|l|\left(\left\|\left[2 \eta_{s} \epsilon\left(\tilde{u}_{h}\right) n\right]_{l}\right\|_{L^{2}(l)}^{2}+\left\|2 k *\left[\epsilon\left(\tilde{u}_{h}\right) n\right]_{l}\right\|_{L^{2}(l)}^{2}\right) \\
& +\left\|i_{h} g-g\right\|_{L^{2}(K)}^{2}+\left\|k *\left(i_{h} \epsilon\left(\tilde{u}_{h}\right)-\epsilon\left(\tilde{u}_{h}\right)\right)\right\|_{L^{2}(K)}^{2} \\
& \left.+\int_{\Omega}\left\|\frac{\partial \tilde{q}_{h}^{D}}{\partial t}-\frac{1}{2 \lambda} \tilde{q}_{h}^{D}-\left(\nabla \tilde{u}_{h}\right) q^{S}-w\right\|_{L^{2}(K)}^{2}\right\}+\left\|u_{0}-i_{h} u_{0}\right\|_{L^{2}(K)}^{2},
\end{aligned}
$$


almost everywhere in $[0, T] \times \Omega$, where $\left(\tilde{u}_{h}, \tilde{q}_{h}^{D}\right)=\mathrm{T}_{h}\left(f_{1}, u_{0}, f_{2}, w\right)$ is given by (3.6). We recall that $i_{h}$ denotes indifferently the $L^{2}$-projections onto $V_{h}$ or $M_{h}$ and that given $q^{S} \in L^{\gamma}\left(\Omega ; h^{\mu}([0, T])\right)$ and $w \in L^{\gamma}\left(h^{\mu}\left(W^{1, r}\right)\right)$, the function $g$ present in the error estimator $\mu_{K}$ is defined for $t \in[0, T]$ by

$$
g(t)=\frac{\eta_{p}}{\lambda} \int_{0}^{t} \mathrm{e}^{-\frac{t-s}{2 \lambda}} \mathbb{E}\left(w(s) \otimes q^{S}(t)+q^{S}(t) \otimes w(s)\right) \mathrm{d} s .
$$

Let T : $Y \times U \times W \rightarrow X$ be the continuous linear operator defined by (3.8). The following a posteriori error estimate holds for the operator $\mathrm{T}-\mathrm{T}_{h}$.

Lemma 4.1. There exists $C$ such that for all $h>0$ and for all $\left(f_{1}, u_{0}, f_{2}, w\right) \in Y \times U \times W$ we have

$$
\left\|\left(\mathrm{T}-\mathrm{T}_{h}\right)\left(f_{1}, u_{0}, f_{2}, w\right)\right\|_{X_{h}} \leq C \sum_{K \in \mathcal{T}_{h}} \mu_{K}^{2}\left(f_{1}, u_{0}, f_{2}, w\right) .
$$

The proof of Lemma (4.1) is provided in Appendix B.

We are now in position to state a posteriori error estimates for the solution of (3.2).

Theorem 4.2. Let $y=\left(f, u_{0}\right) \in Y$ be sufficiently small and let $x(y)=\left(u(y), q^{D}(y)\right) \in X$ be the solution of (2.3)-(2.8). Let $h$ be sufficiently small and let $x_{h}(y)=\left(u_{h}(y), q_{h}^{D}(y)\right) \in X_{h}$ be the solution of (3.2). Then there exists a constant $C$ independent of $h$ and $y$ such that the following a posteriori error estimate holds

$$
\left\|x(y)-x_{h}(y)\right\|_{X_{h}} \leq C\left(\sum_{K \in \mathcal{T}_{h}} \mu_{K}^{2}\left(f, u_{0}, S_{c}\left(x_{h}(y)\right), S_{d}\left(x_{h}(y)\right)\right)\right)^{1 / 2} .
$$

Proof. We recall that $x=x(y) \in X$ and $x_{h}=x_{h}(y) \in X_{h}$ satisfy

$$
x=\mathrm{T}\left(y, S_{c}(x), S_{d}(x)\right), \quad x_{h}=\mathrm{T}_{h}\left(y, S_{c}\left(x_{h}\right), S_{d}\left(x_{h}\right)\right),
$$

where the operators $S_{c}$ and $S_{d}$ are defined by (3.3) and (3.4) respectively. It follows

$$
\begin{aligned}
x-x_{h} & =\mathrm{T}\left(y, S_{c}(x), S_{d}(x)\right)-\mathrm{T}_{h}\left(y, S_{c}\left(x_{h}\right), S_{d}\left(x_{h}\right)\right) \\
& =\mathrm{T}\left(0, S_{c}(x)-S_{c}\left(x_{h}\right), S_{d}(x)-S_{d}\left(x_{h}\right)\right)+\left(\mathrm{T}-\mathrm{T}_{h}\right)\left(y, S_{c}\left(x_{h}\right), S_{d}\left(x_{h}\right)\right) .
\end{aligned}
$$

We now bound the first term in the right hand side of the above equation. Let $\bar{x}=\left(\bar{u}, \bar{q}^{D}\right)$ be defined by

$$
\bar{x}=\mathrm{T}\left(0, S_{c}(x)-S_{c}\left(x_{h}\right), S_{d}(x)-S_{d}\left(x_{h}\right)\right),
$$

then there exists $\bar{p} \in L^{2}\left(0, T ; L_{0}^{2}(D)\right)$ such that

$$
\left(\bar{u}, \bar{q}^{D}\right) \in L^{\infty}\left(0, T ; L^{2}(D)\right) \cap L^{2}\left(0, T ; H_{0}^{1}(D)\right) \times L^{2}\left(\Omega ; L^{\infty}\left(0, T ; L^{2}(D)\right)\right)
$$

and satisfies

$$
\begin{aligned}
\left.\rho \frac{\partial \bar{u}}{\partial t}-2 \eta_{s} \nabla \cdot \epsilon(\bar{u})+\nabla \bar{p}-\frac{\eta_{p}}{\lambda} \nabla \cdot \mathbb{E}\left(\bar{q}^{D} \otimes q^{S}+q^{S} \otimes \bar{q}^{D}\right)\right) & =\frac{\eta_{p}}{\lambda}\left(S_{c}(x)-S_{c}\left(x_{h}\right)\right), \\
\nabla \cdot \bar{u} & =0, \\
\frac{\partial \bar{q}^{D}}{\partial t}-\frac{1}{2 \lambda} \bar{q}^{D}-(\nabla \bar{u}) q^{S} & =S_{d}(x)-S_{d}\left(x_{h}\right), \\
\bar{u}(0) & =0, \\
\bar{q}^{D}(0) & =0 .
\end{aligned}
$$


Recalling that $\int_{0}^{T}(k * \epsilon(\bar{u}), \epsilon(\bar{u})) \geq 0$ (Lem. 1 in [66]) and using a classical method to obtain energy estimate for the Stokes system, there exists a constant $C$ independent of $h, x$ and $x_{h}$ such that

$$
\left\|\bar{u}, \bar{q}^{D}\right\|_{X_{h}} \leq C\left(\left\|S_{c}(x)-S_{c}\left(x_{h}\right)\right\|_{L^{2}\left(L^{2}\right)}+\left\|S_{d}(x)-S_{d}\left(x_{h}\right)\right\|_{L^{2}\left(L^{2}\left(L^{2}\right)\right)}\right) .
$$

Now we have

$$
\begin{aligned}
S_{d}(x)-S_{d}\left(x_{h}\right) & =(\nabla u) q^{D}-\left(\nabla u_{h}\right) q_{h}^{D} \\
& =\left(\nabla u-u_{h}\right) q^{D}+\left(\nabla u_{h}\right)\left(q^{D}-q_{h}^{D}\right),
\end{aligned}
$$

so that

$$
\begin{aligned}
\left\|S_{d}(x)-S_{d}\left(x_{h}\right)\right\|_{L^{2}\left(L^{2}\left(L^{2}\right)\right)} & \leq\left\|\nabla\left(u-u_{h}\right)\right\|_{L^{2}\left(L^{2}\right)}\left\|q^{D}\right\|_{L^{2}\left(L^{\infty}\left(L^{\infty}\right)\right)}+\left\|\nabla u_{h}\right\|_{L^{2}\left(L^{\infty}\right)}\left\|q^{D}-q_{h}^{D}\right\|_{L^{2}\left(L^{\infty}\left(L^{2}\right)\right)} \\
& \leq\left\|x-x_{h}\right\|_{X_{h}}\left(\left\|q^{D}\right\|_{L^{2}\left(L^{\infty}\left(L^{\infty}\right)\right)}^{2}+\left\|\nabla u_{h}\right\|_{L^{2}\left(L^{\infty}\right)}^{2}\right)^{1 / 2} .
\end{aligned}
$$

Let us now bound $\left\|\nabla u_{h}\right\|_{L^{2}\left(L^{\infty}\right)}$. We have

$$
\begin{aligned}
\left\|\nabla u_{h}\right\|_{L^{2}\left(L^{\infty}\right)} & \leq\left\|\nabla\left(u-u_{h}\right)\right\|_{L^{2}\left(L^{\infty}\right)}+\|\nabla u\|_{L^{2}\left(L^{\infty}\right)} \\
& \leq\left\|\nabla\left(u-i_{h} u\right)\right\|_{L^{2}\left(L^{\infty}\right)}+\left\|\nabla\left(i_{h} u-u_{h}\right)\right\|_{L^{2}\left(L^{\infty}\right)}+\|\nabla u\|_{L^{2}\left(L^{\infty}\right)},
\end{aligned}
$$

where $i_{h}$ is the $L^{2}$-projection onto $V_{h}$. Standard interpolation estimates, an inverse estimate, and a Sobolev imbedding theorem can be used to obtain

$$
\left\|\nabla u_{h}\right\|_{L^{2}\left(L^{\infty}\right)} \leq C\left(\|u\|_{L^{2}\left(W^{2, r}\right)}+\frac{1}{h}\left\|\nabla\left(i_{h} u-u_{h}\right)\right\|_{L^{2}\left(L^{2}\right)}\right),
$$

with $C$ independent of $x, x_{h}$ and $h$. Using estimate (3.31) we find

$$
\left\|\nabla u_{h}\right\|_{L^{2}\left(L^{\infty}\right)} \leq C\left(\|y\|_{Y}+\|x\|_{X}+\|x\|_{X}^{2}\right)
$$

so that

$$
\left\|S_{d}(x)-S_{d}\left(x_{h}\right)\right\|_{L^{2}\left(L^{2}\left(L^{2}\right)\right.} \leq C\left(\|y\|_{Y}+\|x\|_{X}+\|x\|_{X}^{2}\right)
$$

$C$ being independent of $h, y$ and $x$. Similarly, one obtains

$$
\left\|S_{c}(x)-S_{c}\left(x_{h}\right)\right\|_{L^{2}\left(L^{2}\right)} \leq C\left(\|y\|_{Y}+\|x\|_{X}+\|x\|_{X}^{2}\right) .
$$

Thus, we have shown that

$$
\left\|\mathrm{T}\left(0, S_{c}(x)-S_{c}\left(x_{h}\right), S_{d}(x)-S_{d}\left(x_{h}\right)\right)\right\|_{X_{h}} \leq C\left\|x-x_{h}\right\|_{X_{h}}\left(\|y\|_{Y}+\|x\|_{X}+\|x\|_{X}^{2}\right),
$$

where $C$ is independent of $h, y$ and $x$.

In order to bound the second term in the right hand side of (4.1), we use Lemma 4.1. There exists a constant $C$ independent of $h, y$ and $x$ such that for $h$ sufficiently small

$$
\left\|\left(\mathrm{T}-\mathrm{T}_{h}\right)\left(f, u_{0}, S_{c}\left(x_{h}\right), S_{d}\left(x_{h}\right)\right)\right\|_{X_{h}} \leq C\left(\sum_{K \in \mathcal{T}_{h}} \mu_{K}^{2}\left(f, u_{0}, S_{c}\left(x_{h}\right), S_{d}\left(x_{h}\right)\right)\right)^{1 / 2} .
$$


Finally, using the above estimate and estimate (4.2) in (4.1) one obtains

$$
\left\|x-x_{h}\right\|_{X_{h}} \leq C\left(\left\|x-x_{h}\right\|_{X_{h}}\left(\|y\|_{Y}+\|x\|_{X}+\|x\|_{X}^{2}\right)+\sum_{K \in \mathcal{T}_{h}} \mu_{K}^{2}\left(f, u_{0}, S_{c}\left(x_{h}(y)\right), S_{d}\left(x_{h}(y)\right)\right)\right) .
$$

From the continuity of the mapping $y \mapsto x(y)$ we conclude the proof for $y \in Y$ sufficiently small.

\section{Numerical RESUlts}

Let $(u, q)$ be the solution of (1.1)-(1.6), let $\left(u_{h}, q_{h}\right)$ be the solution of (1.9). According to Theorem 3.6 and Corollary 3.8 , our goal is now to check numerically that

$$
\left(\int_{0}^{T}\left\|\nabla\left(u-u_{h}\right)\right\|_{L^{2}(D)}^{2}\right)^{1 / 2}+\sup _{0 \leq t \leq T}\left\|\mathbb{E}(q \otimes q)-\mathbb{E}\left(q_{h} \otimes q_{h}\right)\right\|_{L^{2}(D)}=O(h),
$$

for a simple test case. For this purpose we consider the fully discretized scheme corresponding to (1.9). Then, we check convergence with respect to the mesh size $h$ when the error due to the time step and that due to the Monte Carlo method is negligible.

Following $[13,18]$, we use equilibrium control variates in order to reduce the variance due to the Monte Carlo method. This corresponds to computing $u, p, q$ and $q^{S}$ (the so-called control variable corresponding to the dumbbells elongations at equilibrium) such that

$$
\begin{aligned}
& \mathrm{d} q-\left((\nabla u) q-\frac{1}{2 \lambda} q\right) \mathrm{d} t-\frac{1}{\sqrt{\lambda}} \mathrm{d} B=0, \\
& \mathrm{~d} q^{S}+\frac{1}{2 \lambda} q^{S} \mathrm{~d} t-\frac{1}{\sqrt{\lambda}} \mathrm{d} B=0, \\
& \rho \frac{\partial u}{\partial t}-\nabla \cdot\left(2 \eta_{s} \epsilon(u)+\frac{\eta_{p}}{\lambda}\left(\mathbb{E}(q \otimes q)-\mathbb{E}\left(q^{S} \otimes q^{S}\right)\right)\right)+\nabla p=f,
\end{aligned}
$$

and using the same random numbers for $q$ and $q^{S}$. The reader should note that $q^{S}=q^{S}(t, \omega)$ only. Let $P$ be the number of mesh vertices, $\varphi_{i}, i=1, . ., P$, be the usual hat functions attached to the vertices. Let $N$ be the number of time steps, let $\tau=T / N$ be the time step, $t^{n}=n \tau, n=0, \ldots, N$. Finally, let $J$ be the number of realizations of the Monte-Carlo method. At time $t=0$, we set the initial dumbbells elongations

$$
q_{h, j}^{0}(x)=\sum_{i=1}^{P} q_{j}^{0} \varphi_{i}(x) \quad \forall x \in D
$$

where the two components of $q_{j}^{0}, j=1, \ldots, J$, are independent $\mathcal{N}(0,1)$ random variables. We also set the initial control variates $q_{j}^{S, 0}$ to the same random variables, that is $q_{j}^{S, 0}=q_{j}^{0}, j=1, \ldots, J$, and the initial velocity $u_{h}^{0}=i_{h} u_{0} \in V_{h}$. Then, for $n=0, \ldots, N-1$, we proceed as follows. Assume that $u_{h}^{n} \in V_{h}, q_{h, j}^{n} \in R_{h}, j=1, \ldots, J$, and that the random variables $q_{j}^{S, n}, j=1, \ldots, J$, are known. We first compute $u_{h}^{n+1} \in V_{h}$ and $p_{h}^{n+1} \in Q_{h}$ such that

$$
\begin{aligned}
\rho\left(\frac{u_{h}^{n+1}-u_{h}^{n}}{\tau}, v_{h}\right)+2 \eta_{s}\left(\epsilon\left(u_{h}^{n+1}\right), \epsilon\left(v_{h}\right)\right)- & \left(p_{h}^{n+1}, \nabla \cdot v_{h}\right)+\left(\nabla \cdot u_{h}^{n+1}, s_{h}\right)+\sum_{K \in \mathcal{T}_{h}} \frac{\alpha h_{K}^{2}}{2 \eta_{p}}\left(\nabla p_{h}^{n+1}, \nabla s_{h}\right)_{K} \\
& =\left(f\left(t^{n+1}\right), v_{h}\right)-\frac{\eta_{p}}{\lambda}\left(\frac{1}{J} \sum_{j=1}^{J}\left(q_{h, j}^{n} \otimes q_{h, j}^{n}-q_{j}^{S, n} \otimes q_{j}^{S, n}\right), \epsilon\left(v_{h}\right)\right),
\end{aligned}
$$


for all $\left(v_{h}, r_{h}\right) \in V_{h} \times R_{h}$. Then, the dumbbells elongations are updated

$$
\begin{aligned}
& \left(1+\frac{\tau}{2 \lambda}\right)\left(q_{h, j}^{n+1}, r_{h}\right)=\left(q_{h, j}^{n}, r_{h}\right)+\tau\left(\nabla u_{h}^{n+1} q_{h, j}^{n}, r_{h}\right)+\sqrt{\frac{\tau}{\lambda}} B_{j}^{n}, \\
& \left(1+\frac{\tau}{2 \lambda}\right) q_{j}^{S, n+1}=q_{j}^{S, n}+\sqrt{\frac{\tau}{\lambda}} B_{j}^{n} .
\end{aligned}
$$

Here the two components of $B_{j}^{n}, j=1, \ldots, J$, are independent $\mathcal{N}(0,1)$ random variables. As usual, the mass matrices are lumped so that the computation of $q_{h, j}^{n+1}$ becomes explicit. Following the theoretical results of [38] (one-dimensional shear flow) and the numerical results reported in [13], it is expected that

$$
\left(\sum_{n=0}^{N} \tau\left\|\nabla\left(u\left(t^{n}\right)-u_{h}^{n}\right)\right\|_{L^{2}(D)}^{2}\right)^{1 / 2}+\max _{0 \leq n \leq N}\left\|\mathbb{E}\left(q\left(t^{n}\right) \otimes q\left(t^{n}\right)\right)-\frac{1}{J} \sum_{j=1}^{J} q_{h, j}^{n} \otimes q_{h, j}^{n}\right\|_{L^{2}(D)}=O\left(h+\tau+\frac{1}{\sqrt{J}}\right) .
$$

Therefore, when $\tau=O\left(h^{2}\right)$ and $J=O\left(h^{-4}\right)$, then the error due to time discretization and the error due to the Monte Carlo method should be negligeable, so that $O(h)$ convergence should be observed.

The following simple test case is considered. Let $D=(0,1)^{2}$ be the unit square, the velocity field $u$ is defined by

$$
u\left(x_{1}, x_{2}\right)=\left(\begin{array}{l}
u^{1}\left(x_{2}\right)=e^{x_{2}} \\
u^{2}\left(x_{1}\right)=e^{x_{1}}
\end{array}\right) \quad \forall\left(x_{1}, x_{2}\right) \in D
$$

Setting

$$
\sigma\left(x_{1}, x_{2}, t\right)=\left(\begin{array}{ll}
\sigma^{11} & \sigma^{12} \\
\sigma^{12} & \sigma^{22}
\end{array}\right)
$$

the simplified Oldroyd-B constitutive relationship for the extra-stress $\sigma$-equation (0.5) without the convective term - becomes

$$
\begin{aligned}
& \sigma^{11}+\lambda\left(\frac{\partial \sigma^{11}}{\partial t}-2\left(u^{1}\right)^{\prime} \sigma^{12}\right)=0 \\
& \sigma^{12}+\lambda\left(\frac{\partial \sigma^{12}}{\partial t}-\left(u^{2}\right)^{\prime} \sigma^{11}-\left(u^{1}\right)^{\prime} \sigma^{22}\right)=\eta_{p}\left(\left(u^{1}\right)^{\prime}+\left(u^{2}\right)^{\prime}\right) \\
& \sigma^{22}+\lambda\left(\frac{\partial \sigma^{22}}{\partial t}-2\left(u^{2}\right)^{\prime} \sigma^{12}\right)=0 .
\end{aligned}
$$


Choosing $\sigma(0)=0$, a simple but tedious calculation yields to the following formula for the extra-stress

$$
\begin{aligned}
& \sigma^{11}=\eta_{p} \frac{\left(u^{1}\right)^{\prime}+\left(u^{2}\right)^{\prime}}{1-4 \lambda^{2}\left(u^{1}\right)^{\prime}\left(u^{2}\right)^{\prime}}\left(2 \lambda\left(u^{1}\right)^{\prime}-\frac{1}{2} \sqrt{\frac{\left(u^{1}\right)^{\prime}}{\left(u^{2}\right)^{\prime}}}\left(\left(1+2 \lambda \sqrt{\left(u^{1}\right)^{\prime}\left(u^{2}\right)^{\prime}}\right) \mathrm{e}^{-\frac{t}{\lambda}\left(1-2 \lambda \sqrt{\left(u^{1}\right)^{\prime}\left(u^{2}\right)^{\prime}}\right)}\right.\right. \\
& \left.\left.-\left(1-2 \lambda \sqrt{\left(u^{1}\right)^{\prime}\left(u^{2}\right)^{\prime}}\right) \mathrm{e}^{-\frac{t}{\lambda}\left(1+2 \lambda \sqrt{\left(u^{1}\right)^{\prime}\left(u^{2}\right)^{\prime}}\right)}\right)\right), \\
& \sigma^{12}=\eta_{p} \frac{\left(u^{1}\right)^{\prime}+\left(u^{2}\right)^{\prime}}{1-4 \lambda^{2}\left(u^{1}\right)^{\prime}\left(u^{2}\right)^{\prime}}\left(1-\frac{1}{2}\left(\left(1+2 \lambda \sqrt{\left(u^{1}\right)^{\prime}\left(u^{2}\right)^{\prime}}\right) \mathrm{e}^{-\frac{t}{\lambda}\left(1-2 \lambda \sqrt{\left(u^{1}\right)^{\prime}\left(u^{2}\right)^{\prime}}\right)}\right.\right. \\
& \left.\left.+\left(1-2 \lambda \sqrt{\left(u^{1}\right)^{\prime}\left(u^{2}\right)^{\prime}}\right) \mathrm{e}^{-\frac{t}{\lambda}\left(1+2 \lambda \sqrt{\left(u^{1}\right)^{\prime}\left(u^{2}\right)^{\prime}}\right)}\right)\right), \\
& \sigma^{22}=\eta_{p} \frac{\left(u^{1}\right)^{\prime}+\left(u^{2}\right)^{\prime}}{1-4 \lambda^{2}\left(u^{1}\right)^{\prime}\left(u^{2}\right)^{\prime}}\left(2 \lambda\left(u^{2}\right)^{\prime}-\frac{1}{2} \sqrt{\frac{\left(u^{2}\right)^{\prime}}{\left(u^{1}\right)^{\prime}}}\left(\left(1+2 \lambda \sqrt{\left(u^{1}\right)^{\prime}\left(u^{2}\right)^{\prime}}\right) \mathrm{e}^{-\frac{t}{\lambda}\left(1-2 \lambda \sqrt{\left(u^{1}\right)^{\prime}\left(u^{2}\right)^{\prime}}\right)}\right.\right. \\
& \left.\left.-\left(1-2 \lambda \sqrt{\left(u^{1}\right)^{\prime}\left(u^{2}\right)^{\prime}}\right) \mathrm{e}^{-\frac{t}{\lambda}\left(1+2 \lambda \sqrt{\left(u^{1}\right)^{\prime}\left(u^{2}\right)^{\prime}}\right)}\right)\right) \text {, }
\end{aligned}
$$

whenever $\lambda<1 / \sqrt{4 \max \left|\left(u^{1}\right)^{\prime}\left(u^{2}\right)^{\prime}\right|}=\frac{1}{2 \mathrm{e}} \simeq 0.184$. Using $(0.4)$, that is

$$
\sigma^{11}=\frac{\eta_{p}}{\lambda}\left(\mathbb{E}\left(q^{1} q^{1}\right)-1\right), \quad \sigma^{12}=\frac{\eta_{p}}{\lambda}\left(\mathbb{E}\left(q^{1} q^{2}\right)\right), \quad \sigma^{22}=\frac{\eta_{p}}{\lambda}\left(\mathbb{E}\left(q^{2} q^{2}\right)-1\right)
$$

where $q=\left(\begin{array}{c}q^{1} \\ q^{2}\end{array}\right)$, we therefore obtain explicit formula for $\mathbb{E}\left(q^{1} q^{1}\right), \mathbb{E}\left(q^{1} q^{2}\right)$ and $\mathbb{E}\left(q^{2} q^{2}\right)$.

The viscosities are $\eta_{s}=\eta_{p}=1$, the elastic relaxation time is $\lambda=0.1$ and the GLS stabilization parameter in (1.9) is $\alpha=0.01$. In Table 1 , we have reported the error for the velocity and extra-stress defined by

$$
\begin{aligned}
& e_{u^{i}}=\left(\sum_{n=0}^{N} \tau\left\|\nabla\left(u^{i}\left(t^{n}\right)-u_{h}^{i, n}\right)\right\|_{L^{2}(D)}^{2}\right)^{1 / 2} i=1,2, \\
& e_{\sigma^{k l}}=\frac{\eta_{p}}{\lambda} \max _{0 \leq n \leq N}\left\|\mathbb{E}\left(q^{k}\left(t^{n}\right) q^{l}\left(t^{n}\right)\right)-\frac{1}{J} \sum_{j=1}^{J} q_{h, j}^{k, n} q_{h, j}^{l, n}\right\|_{L^{2}(D)} \quad k, l=1,2,
\end{aligned}
$$

where we have set

$$
u_{h}^{n}=\left(\begin{array}{c}
u_{h}^{1, n} \\
u_{h}^{2, n}
\end{array}\right) \quad \text { and } \quad q_{h, j}^{n}=\left(\begin{array}{c}
q_{h, j}^{1, n} \\
q_{h, j}^{2, n}
\end{array}\right) .
$$

The results reported in Table 1 are given with two digits. They correspond to averages over 30 runs. The $95 \%$ confidence intervals are reported that is average value \pm twice the root mean square. Clearly, the error is $O(h)$ which corresponds to theoretical results. It should be noted that the memory required to perform the last computation corresponding to the last row in Table 1 is $1.7 \mathrm{~Gb}$ (400 vertices times 256000 dumbbells thus $400 \times 2 \times 256000 \times 8=1.64 \mathrm{~Gb}$ ). Therefore, performing a computation with a $40 \times 40$ mesh and 16 times more dumbbells would require more than 64 Gb memory. This is possible only with a parallel, distributed memory machine and is not within the scope of this contribution. 
TABLE 1. Error with respect to mesh size $h$, with $95 \%$ confidence intervals corresponding to 30 runs.

\begin{tabular}{|c|c|c|c|c|c|c|c|c|c|}
\hline Mesh & $h$ & $\tau$ & $N$ & $J$ & $e_{u^{1}}$ & $e_{u^{2}}$ & $e_{\sigma^{11}}$ & $e_{\sigma^{12}}$ & $e_{\sigma^{22}}$ \\
\hline $5 \times 5$ & 0.2 & 0.01 & 50 & 1000 & $0.073 \pm 0.000$ & $0.073 \pm 0.000$ & $0.36 \pm 0.18$ & $0.46 \pm 0.17$ & $0.36 \pm 0.17$ \\
\hline $10 \times 10$ & 0.1 & 0.0025 & 200 & 16000 & $0.037 \pm 0.000$ & $0.037 \pm 0.000$ & $0.13 \pm 0.04$ & $0.19 \pm 0.03$ & $0.13 \pm 0.03$ \\
\hline $20 \times 20$ & 0.05 & 0.000625 & 800 & 256000 & $0.018 \pm 0.000$ & $0.018 \pm 0.000$ & $0.061 \pm 0.006$ & $0.089 \pm 0.003$ & $0.060 \pm 0.005$ \\
\hline
\end{tabular}

Acknowledgements. A.B. thanks Claude Le Bris and Tony Lelièvre for interesting discussions during a short stay at CERMICS, École nationale des ponts et chaussées, Paris. A.B. and M.P. thank Alexei Lozinski and Jacques Rappaz for the permanent interest they have shown for this research.

\section{Appendix A. Proof of (3.13) in Lemma 3.3}

Let

$$
\begin{array}{lll}
e_{u}=\tilde{u}-\tilde{u}_{h}=\Pi_{u}+C_{u}, & \Pi_{u}=\tilde{u}-i_{h} \tilde{u}, & C_{u}=i_{h} \tilde{u}-\tilde{u}_{h}, \\
e_{p}=\tilde{p}-\tilde{p}_{h}=\Pi_{p}+C_{p}, & \Pi_{p}=\tilde{p}-i_{h} \tilde{p}, & C_{p}=i_{h} \tilde{p}-\tilde{p}_{h},
\end{array}
$$

where $\left(\tilde{u}_{h}, \tilde{p}_{h}\right)$ solve $(3.9)$ and $(\tilde{u}, \tilde{p})$ solve $(2.15)$. Using the triangle inequality we have

$$
\left\|e_{u}\right\|_{L^{2}\left(H^{1}\right)} \leq\left\|\Pi_{u}\right\|_{L^{2}\left(H^{1}\right)}+\left\|C_{u}\right\|_{L^{2}\left(H^{1}\right)} .
$$

Using classical interpolation results, we obtain

$$
\left\|\Pi_{u}\right\|_{L^{2}\left(H^{1}\right)} \leq C h\|\tilde{u}\|_{L^{2}\left(H^{2}\right)} .
$$

We now estimate $\left\|C_{u}\right\|_{L^{2}\left(H^{1}\right)}$. The solution of (2.15) satisfies

$\rho\left(\frac{\partial \tilde{u}}{\partial t}, v_{h}\right)+2 \eta_{s}\left(\epsilon(\tilde{u}), \epsilon\left(v_{h}\right)\right)-\left(\tilde{p}, \nabla \cdot v_{h}\right)+2\left(k * \epsilon(\tilde{u}), \epsilon\left(v_{h}\right)\right)-\left(f_{1}, v_{h}\right)+\left(\nabla \cdot \tilde{u}, s_{h}\right)+\left(\frac{\eta_{p}}{\lambda} f_{2}-g, \epsilon\left(v_{h}\right)\right)=0$

for all $\left(v_{h}, s_{h}\right) \in V_{h} \times Q_{h}$. Subtracting (3.9) to the above equation, it follows that

$$
\begin{aligned}
\rho\left(\frac{\partial e_{u}}{\partial t}, v_{h}\right)+2 \eta_{s}\left(\epsilon\left(e_{u}\right), \epsilon\left(v_{h}\right)\right)- & \left(e_{p}, \nabla \cdot v_{h}\right)+2\left(k *\left(\epsilon(\tilde{u})-i_{h} \epsilon\left(\tilde{u}_{h}\right)\right), \epsilon\left(v_{h}\right)\right) \\
& +\left(\nabla \cdot e_{u}, r_{h}\right)+\sum_{K \in \mathcal{T}_{h}} \frac{\alpha h_{K}^{2}}{2 \eta_{p}}\left(\nabla e_{p}-\nabla \tilde{p}, \nabla r_{h}\right)_{K}-\left(g-i_{h} g, \epsilon\left(v_{h}\right)\right)=0,
\end{aligned}
$$

for all $\left(v_{h}, r_{h}\right) \in V_{h} \times Q_{h}$. On the other hand, from the definition of $i_{h}$ (the $L^{2}$ projection onto the finite element spaces), $C_{u}$ and $\Pi_{u}$, we have

$$
\begin{aligned}
\left(k * i_{h} \epsilon\left(C_{u}\right), i_{h} \epsilon\left(C_{u}\right)\right) & =\left(k * i_{h} \epsilon\left(C_{u}\right), \epsilon\left(C_{u}\right)\right) \\
& =\left(k *\left(\epsilon(\tilde{u})-i_{h} \epsilon\left(\tilde{u}_{h}\right)\right)+k *\left(i_{h} \epsilon(\tilde{u})-\epsilon(\tilde{u})\right)-k * i_{h} \epsilon\left(\Pi_{u}\right), \epsilon\left(C_{u}\right)\right) .
\end{aligned}
$$


Hence, we obtain

$$
\begin{array}{r}
\rho\left(\frac{\partial C_{u}}{\partial t}, C_{u}\right)+2 \eta_{s}\left(\epsilon\left(C_{u}\right), \epsilon\left(C_{u}\right)\right)-\left(C_{p}, \nabla \cdot C_{u}\right)+2\left(k * i_{h} \epsilon\left(C_{u}\right), i_{h} \epsilon\left(C_{u}\right)\right)+\left(\nabla \cdot C_{u}, C_{p}\right)+\sum_{K \in \mathcal{T}_{h}} \frac{\alpha h_{K}^{2}}{2 \eta_{p}}\left(\nabla C_{p}, \nabla C_{p}\right)_{K} \\
=\rho\left(\frac{\partial\left(e_{u}-\Pi_{u}\right)}{\partial t}, C_{u}\right)+2 \eta_{s}\left(\epsilon\left(e_{u}-\Pi_{u}\right), \epsilon\left(C_{u}\right)\right)-\left(e_{p}-\Pi_{p}, \nabla \cdot C_{u}\right) \\
+2\left(k *\left(\epsilon(\tilde{u})-i_{h} \epsilon\left(\tilde{u}_{h}\right)\right), \epsilon\left(C_{u}\right)\right)-2\left(k *\left(\epsilon(\tilde{u})-i_{h} \epsilon(\tilde{u})\right), \epsilon\left(C_{u}\right)\right)-2\left(k * i_{h} \epsilon\left(\Pi_{u}\right), \epsilon\left(C_{u}\right)\right) \\
+\left(\nabla \cdot\left(e_{u}-\Pi_{u}\right), C_{p}\right)+\sum_{K \in \mathcal{T}_{h}} \frac{\alpha h_{K}^{2}}{2 \eta_{p}}\left(\nabla\left(e_{p}-\Pi_{p}\right), \nabla C_{p}\right)_{K}(\mathrm{~A} .2)
\end{array}
$$

From the definition of $i_{h}$ again, we obviously have

$$
\left(\frac{\partial \Pi_{u}}{\partial t}, C_{u}\right)=0
$$

so that, using (A.1), (A.2) yields

$$
\begin{aligned}
\rho\left(\frac{\partial C_{u}}{\partial t}, C_{u}\right)+ & 2 \eta_{s}\left(\epsilon\left(C_{u}\right), \epsilon\left(C_{u}\right)\right)+\sum_{K \in \mathcal{T}_{h}} \frac{\alpha h_{K}^{2}}{2 \eta_{p}}\left(\nabla C_{p}, \nabla C_{p}\right)_{K}+2\left(k * i_{h} \epsilon\left(C_{u}\right), i_{h} \epsilon\left(C_{u}\right)\right) \\
& =-2 \eta_{s}\left(\epsilon\left(\Pi_{u}\right), \epsilon\left(C_{u}\right)\right)+\left(\Pi_{p}, \nabla \cdot C_{u}\right)-\left(\nabla \cdot\left(\Pi_{u}\right), C_{p}\right)-\sum_{K \in \mathcal{T}_{h}} \frac{\alpha h_{K}^{2}}{2 \eta_{p}}\left(\nabla \Pi_{p}, \nabla C_{p}\right)_{K} \\
& +\sum_{K \in \mathcal{T}_{h}} \frac{\alpha h_{K}^{2}}{2 \eta_{p}}\left(\nabla \tilde{p}, \nabla C_{p}\right)_{K}+2\left(k *\left(\epsilon(\tilde{u})-i_{h} \epsilon(\tilde{u})\right), \epsilon\left(C_{u}\right)\right)+2\left(k * i_{h} \epsilon\left(\Pi_{u}\right), \epsilon\left(C_{u}\right)\right)+\left(g-i_{h} g, \epsilon\left(C_{u}\right)\right) \\
& =I_{1}+\cdots+I_{8} . \quad(\mathrm{A} .3)
\end{aligned}
$$

It now remains to bound the terms $I_{1}, \ldots, I_{8}$ in the above equality. Using Cauchy-Schwarz and Young's inequalities, we have

$$
\begin{aligned}
I_{1}= & -2 \eta_{s}\left(\epsilon\left(\Pi_{u}\right), \epsilon\left(C_{u}\right)\right) \\
& \leq 2 \eta_{s}\left\|\epsilon\left(\Pi_{u}\right)\right\|_{L^{2}(D)}\left\|\epsilon\left(C_{u}\right)\right\|_{L^{2}(D)} \\
& \leq 5 \eta_{s}\left\|\epsilon\left(\Pi_{u}\right)\right\|_{L^{2}(D)}^{2}+\frac{\eta_{s}}{5}\left\|\epsilon\left(C_{u}\right)\right\|_{L^{2}(D)}^{2}
\end{aligned}
$$

Similarly, we have

$$
\begin{aligned}
I_{2}=\left(\Pi_{p}, \nabla \cdot C_{u}\right) & \leq \frac{5}{4 \eta_{s}}\left\|\Pi_{p}\right\|_{L^{2}(D)}^{2}+\frac{\eta_{s}}{5}\left\|\nabla \cdot C_{u}\right\|_{L^{2}(D)}^{2} \\
& \leq \frac{5}{4 \eta_{s}}\left\|\Pi_{p}\right\|_{L^{2}(D)}^{2}+\frac{\eta_{s}}{5}\left\|\epsilon\left(C_{u}\right)\right\|_{L^{2}(D)}^{2} .
\end{aligned}
$$

An integration by parts yields, since $\Pi_{u}=0$ on $\partial D$

$$
\begin{aligned}
I_{3}= & \left(\nabla \cdot\left(\Pi_{u}\right), C_{p}\right)=-\left(\Pi_{u}, \nabla C_{p}\right)=-\sum_{K \in \mathcal{T}_{h}}\left(\Pi_{u}, \nabla C_{p}\right)_{K} \\
& \leq \sum_{K \in \mathcal{T}_{h}} \frac{\alpha h_{K}^{2}}{12 \eta_{p}}\left\|\nabla C_{p}\right\|_{L^{2}(K)}^{2}+\sum_{K \in \mathcal{T}_{h}} \frac{3 \eta_{p}}{\alpha h_{K}^{2}}\left\|\Pi_{u}\right\|_{L^{2}(K)}^{2} .
\end{aligned}
$$


Again, Cauchy-Schwarz and Young's inequalities yield

$$
I_{4}=-\sum_{K \in \mathcal{T}_{h}} \frac{\alpha h_{K}^{2}}{2 \eta_{p}}\left(\nabla \Pi_{p}, \nabla C_{p}\right)_{K} \leq \sum_{K \in \mathcal{T}_{h}} \frac{\alpha h_{K}^{2}}{12 \eta_{p}}\left\|\nabla C_{p}\right\|_{L^{2}(K)}^{2}+\frac{3 \alpha h^{2}}{4 \eta_{p}}\left\|\nabla \Pi_{p}\right\|_{L^{2}(D)}^{2}
$$

and

$$
I_{5}=\sum_{K \in \mathcal{T}_{h}} \frac{\alpha h_{K}^{2}}{2 \eta_{p}}\left(\nabla \tilde{p}, \nabla C_{p}\right)_{K} \leq \sum_{K \in \mathcal{T}_{h}} \frac{\alpha h_{K}^{2}}{12 \eta_{p}}\left\|\nabla C_{p}\right\|_{L^{2}(K)}^{2}+\frac{3 \alpha h^{2}}{4 \eta_{p}}\|\nabla \tilde{p}\|_{L^{2}(D)}^{2} .
$$

Since $k \in \mathcal{C}^{\infty}([0, T]) \subset_{>} L^{\infty}([0, T])$, using Cauchy-Schwarz and Young's inequalities yield

$$
I_{6}=-2\left(k *\left(i_{h} \epsilon(\tilde{u})-\epsilon(\tilde{u})\right), \epsilon\left(C_{u}\right)\right) \leq \frac{5}{\eta_{s}}\|k\|_{L^{\infty}(0, T)}^{2}\left\|i_{h} \epsilon(\tilde{u})-\epsilon(\tilde{u})\right\|_{L^{2}(D)}^{2}+\frac{\eta_{s}}{5}\left\|\epsilon\left(C_{u}\right)\right\|_{L^{2}(D)}^{2}
$$

and

$$
I_{7}=-2\left(k * i_{h} \epsilon\left(\Pi_{u}\right), \epsilon\left(C_{u}\right)\right) \leq \frac{5}{\eta_{s}}\|k\|_{L^{\infty}}^{2}\left\|\epsilon\left(\Pi_{u}\right)\right\|_{L^{2}(D)}^{2}+\frac{\eta_{s}}{5}\left\|\epsilon\left(C_{u}\right)\right\|_{L^{2}(D)}^{2},
$$

where in the last inequality we used the stability of $i_{h}: L^{2}(D) \rightarrow L^{2}(D)$. Finally, Cauchy-Schwarz and Young's inequalities again yields

$$
I_{8}=\left(g-i_{h} g, \epsilon\left(C_{u}\right)\right) \leq \frac{5}{4 \eta_{s}}\left\|g-i_{h} g\right\|_{L^{2}(D)}^{2}+\frac{\eta_{s}}{5}\left\|\epsilon\left(C_{u}\right)\right\|_{L^{2}(D)}^{2} .
$$

The above estimates of $I_{1}, \ldots, I_{8}$ in (A.3) yield

$$
\begin{aligned}
\rho\left(\frac{\partial C_{u}}{\partial t}, C_{u}\right)+\frac{1}{2} 2 \eta_{s} & \left(\epsilon\left(C_{u}\right), \epsilon\left(C_{u}\right)\right)+\frac{1}{2} \sum_{K \in \mathcal{T}_{h}} \frac{\alpha h_{K}^{2}}{2 \eta_{p}}\left(\nabla C_{p}, \nabla C_{p}\right)_{K}+2\left(k * i_{h} \epsilon\left(C_{u}\right), i_{h} \epsilon\left(C_{u}\right)\right) \\
& \leq C\left(\left\|\epsilon\left(\Pi_{u}\right)\right\|_{L^{2}(D)}^{2}+\left\|\Pi_{p}\right\|_{L^{2}(D)}^{2}+\sum_{K \in \mathcal{T}_{h}} \frac{1}{h_{K}^{2}}\left\|\Pi_{u}\right\|_{L^{2}(K)}^{2}+h^{2}\left\|\nabla \Pi_{p}\right\|_{L^{2}(D)}^{2}+h^{2}\|\nabla \tilde{p}\|_{L^{2}(D)}^{2}\right)
\end{aligned}
$$

where $C$ depends only on $\rho, \eta_{s}, \eta_{p}, k$ and $\alpha$. Time integration for $0 \leq s \leq T$ yields

$$
\begin{aligned}
& \frac{\rho}{2}\left\|C_{u}(s)\right\|_{L^{2}(D)}^{2}+\eta_{s} \int_{0}^{s}\left\|\epsilon\left(C_{u}\right)\right\|_{L^{2}(D)}^{2}+2 \int_{0}^{s}\left(\left(k * i_{h} \epsilon\left(C_{u}\right)\right)(s), i_{h} \epsilon\left(C_{u}(s)\right)\right) \mathrm{d} s \\
& \leq \frac{\rho}{2}\left\|C_{u}(0)\right\|_{L^{2}(D)}^{2}+C \int_{0}^{s}\left(\left\|\epsilon\left(\Pi_{u}\right)\right\|_{L^{2}(D)}^{2}+\left\|\Pi_{p}\right\|_{L^{2}(D)}^{2}+\left\|\Pi_{\sigma}\right\|_{L^{2}(D)}^{2}\right. \\
& \quad+\sum_{K \in \mathcal{T}_{h}} \frac{1}{h_{K}^{2}}\left\|\Pi_{u}\right\|_{L^{2}(K)}^{2}+h^{2}\left\|\nabla \Pi_{p}\right\|_{L^{2}(D)}^{2}+h^{2}\|\nabla \tilde{p}\|_{L^{2}(D)}^{2} .
\end{aligned}
$$

Using standard interpolation results and (3.14), we obtain

$$
\left\|\epsilon\left(C_{u}\right)\right\|_{L^{2}\left(L^{2}\right)}^{2} \leq C h^{2}\left(\|\tilde{u}\|_{h^{\mu}\left(W^{2, r}\right)}^{2}+\|\tilde{p}\|_{h^{\mu}\left(W^{1, r}\right)}^{2}+\left\|\nabla u_{0}\right\|_{L^{2}(D)}^{2}+\|g\|_{L^{2}\left(W^{1, r}(D)\right)}^{2}\right),
$$

where $C$ does not depend on $h, f_{1}, f_{2}, u_{0}$ and $g$. Then, since

$$
\|g\|_{L^{2}\left(W^{1, r}\right)} \leq C\left\|q^{S}\right\|_{L^{2}\left(\Omega ; L^{\infty}([0, T])\right.}\|w\|_{L^{2}\left(L^{2}\left(W^{1, r}\right)\right)} \quad \text { and } \quad\left\|\nabla u_{0}\right\|_{L^{2}(D)} \leq C\left\|u_{0}\right\|_{\mathcal{D}_{A_{r}}}
$$

we obtain

$$
\left\|\epsilon\left(C_{u}\right)\right\|_{L^{2}\left(L^{2}\right)} \leq C h\left(\left\|f_{1}, u_{0}\right\|_{Y}+\left\|f_{2}\right\|_{U}+\|w\|_{W}\right),
$$


where $C$ does not depend on $h, f_{1}, f_{2}, u_{0}$ and $w$. Thus

$$
\left\|\epsilon\left(e_{u}\right)\right\|_{L^{2}\left(L^{2}\right)} \leq C h\left(\left\|f_{1}, u_{0}\right\|_{Y}+\left\|f_{2}\right\|_{U}+\|w\|_{W}\right) .
$$

It remains to prove that

$$
\left\|\tilde{q}^{D}-\tilde{q}_{h}^{D}\right\|_{L^{2}\left(L^{\infty}\left(L^{2}\right)\right)} \leq C h\left(\|y\|_{Y}+\left\|f_{2}\right\|_{U}+\|w\|_{W}\right)
$$

where $C$ does not depend on $h, f_{1}, f_{2}, u_{0}$ and $w$. The solution $\left(\tilde{u}, \tilde{q}^{D}\right)=\mathrm{T}\left(y, f_{2}, w\right)$ satisfies for $t \in[0, T]$

$$
\tilde{q}^{D}(t)=\int_{0}^{t} e^{-\frac{t-s}{2 \lambda}}\left((\nabla \tilde{u}) q^{S}+w\right) \mathrm{d} s .
$$

Hence, (A.5) follows by substracting (3.10) to the above equation.

\section{Appendix B. Proof of Lemma 4.1}

Let $\left(\tilde{u}, \tilde{q}^{D}\right)=\mathrm{T}\left(f_{1}, u_{0}, f_{2}, w\right)$, where $\left(\tilde{u}, \tilde{q}^{D}, \tilde{p}\right)$ is the solution of $(2.9)-(2.14)$ and let $\left(\tilde{u}_{h}, \tilde{q}_{h}^{D}\right)=\mathrm{T}_{h}\left(f_{1}, u_{0}, f_{2}, w\right)$, where $\left(\tilde{u}_{h}, \tilde{q}_{h}^{D}, \tilde{p}_{h}\right)$ is the solution of (3.6). Let

$$
e_{u}=\tilde{u}-\tilde{u}_{h}, \quad \Pi_{u}=e_{u}-i_{h}^{C} e_{u}, \quad e_{p}=\tilde{p}-\tilde{p}_{h},
$$

where $i_{h}^{C}$ is the Clément's interpolant [23]. Using (2.15) it follows

$$
\begin{aligned}
& \rho\left(\frac{\partial e_{u}}{\partial t}, e_{u}\right)+2 \eta_{s}\left(\epsilon\left(e_{u}\right), \epsilon\left(e_{u}\right)\right)+2 k *\left(\epsilon\left(e_{u}\right), \epsilon\left(e_{u}\right)\right) \\
& \quad=\left(f_{1}-\nabla \tilde{p}, e_{u}\right)-\left(\frac{\eta_{p}}{\lambda} f_{2}+g, \epsilon\left(e_{u}\right)\right)-\rho\left(\frac{\partial \tilde{u}_{h}}{\partial t}, e_{u}\right)-2 \eta_{s}\left(\epsilon\left(\tilde{u}_{h}\right), \epsilon\left(e_{u}\right)\right)-2 k *\left(\epsilon\left(\tilde{u}_{h}\right), \epsilon\left(e_{u}\right)\right),
\end{aligned}
$$

where $g$ is defined by (2.16). The decomposition (B.1) leads to

$$
\begin{aligned}
& \rho\left(\frac{\partial e_{u}}{\partial t}, e_{u}\right)+2 \eta_{s}\left(\epsilon\left(e_{u}\right), \epsilon\left(e_{u}\right)\right)+2 k *\left(\epsilon\left(e_{u}\right), \epsilon\left(e_{u}\right)\right) \\
& \quad=\left(f_{1}-\nabla \tilde{p}, \Pi_{u}\right)-\left(\frac{\eta_{p}}{\lambda} f_{2}+g, \epsilon\left(\Pi_{u}\right)\right)-\rho\left(\frac{\partial \tilde{u}_{h}}{\partial t}, \Pi_{u}\right)-2 \eta_{s}\left(\epsilon\left(\tilde{u}_{h}\right), \epsilon\left(\Pi_{u}\right)\right)-2 k *\left(\epsilon\left(\tilde{u}_{h}\right), \epsilon\left(\Pi_{u}\right)\right) \\
& \quad+\left(f_{1}-\nabla \tilde{p}, i_{h}^{C} e_{u}\right)-\left(\frac{\eta_{p}}{\lambda} f_{2}+g, \epsilon\left(i_{h}^{C} e_{u}\right)\right)-\rho\left(\frac{\partial \tilde{u}_{h}}{\partial t}, i_{h}^{C} e_{u}\right)-2 \eta_{s}\left(\epsilon\left(\tilde{u}_{h}\right), \epsilon\left(i_{h}^{C} e_{u}\right)\right)-2 k *\left(\epsilon\left(\tilde{u}_{h}\right), \epsilon\left(i_{h}^{C} e_{u}\right)\right)
\end{aligned}
$$

Hence, using relation (3.9) and since $\nabla \cdot u=0$, we have after reordering the terms

$$
\begin{aligned}
\rho\left(\frac{\partial e_{u}}{\partial t}, e_{u}\right)+2 \eta_{s} & \left(\epsilon\left(e_{u}\right), \epsilon\left(e_{u}\right)\right)+2 k *\left(\epsilon\left(e_{u}\right), \epsilon\left(e_{u}\right)\right) \\
& =-2 \eta_{s}\left(\epsilon\left(\tilde{u}_{h}\right), \epsilon\left(\Pi_{u}\right)\right)+\left(\tilde{p}_{h}, \nabla \cdot \Pi_{u}\right)-2 k *\left(\epsilon\left(\tilde{u}_{h}\right), \epsilon\left(\Pi_{u}\right)\right)-\left(\frac{\eta_{p}}{\lambda} f_{2}+g, \epsilon\left(\Pi_{u}\right)\right) \\
+ & \left(f_{1}, \Pi_{u}\right)-\rho\left(\frac{\partial \tilde{u}_{h}}{\partial t}, \Pi_{u}\right)+\left(e_{p}, \nabla \cdot u_{h}\right)+\left(i_{h} g-g, \epsilon\left(i_{h}^{C} e_{u}\right)\right)+2 k *\left(i_{h} \epsilon\left(\tilde{u}_{h}\right)-\epsilon\left(\tilde{u}_{h}\right), \epsilon\left(i_{h}^{C} e_{u}\right)\right) .
\end{aligned}
$$


We then proceed as in [5,67], integrate by parts on each triangle $K \in \mathcal{T}_{h}$ the first four terms in the right hand side of the above equation and use Cauchy-Schwarz inequalities, interpolation results for Clément's interpolant to obtain

$$
\begin{aligned}
& \rho\left(\frac{\partial e_{u}}{\partial t}, e_{u}\right)+2 \eta_{s}\left(\epsilon\left(e_{u}\right), \epsilon\left(e_{u}\right)\right)+2 k *\left(\epsilon\left(e_{u}\right), \epsilon\left(e_{u}\right)\right) \\
& \leq C\left(\sum_{K \in \mathcal{T}_{h}} \mu_{K}^{2}\left(f_{1}, u_{0}, f_{2}, w\right)\right)^{1 / 2}\left(\left\|e_{p}\right\|_{L^{2}(D)}+\left\|\epsilon\left(e_{u}\right)\right\|_{L^{2}(D)}\right)
\end{aligned}
$$

where $C$ is a constant only depending on the physical domain $D$. Then, a time integration and Young inequalities lead to

$$
\frac{1}{2}\left\|e_{u}(T)\right\|_{L^{2}(D)}^{2}+\eta_{s}\left\|\epsilon\left(e_{u}\right)\right\|_{L^{2}\left(L^{2}\right)}^{2}+\int_{0}^{T} 2 k *\left\|\epsilon\left(e_{u}\right)\right\|_{L^{2}(D)}^{2} \leq C \sum_{K \in \mathcal{T}_{h}} \mu_{K}^{2}\left(f_{1}, u_{0}, f_{2}, w\right)+\frac{1}{2}\left\|e_{p}\right\|_{L^{2}\left(L^{2}\right)}^{2}
$$

where $C$ is a constant independent of $h$.

In order to estimate the last term of the right hand side, let us consider $w \in H^{1}\left(0, T ; H^{-1}(D)\right) \cap L^{2}\left(0, T ; H^{1}(D)\right)$ and $r \in L^{2}\left(0, T ; L^{2}(D)\right)$ a solution of the problem

$$
-\rho\left(\frac{\partial w}{\partial t}, \bar{v}\right)+2 \eta_{s}(\epsilon(w), \epsilon(\bar{v}))+2(k * \epsilon(w), \epsilon(\bar{v}))-(r, \nabla \cdot \bar{v})+(\bar{r}, \nabla \cdot w)=\left(e_{p}, \bar{v}\right),
$$

for all $(\bar{v}, \bar{r}) \in H_{0}^{1}(D) \times L^{2}(D)$. Recalling that $\left.\int_{0}^{T}(k * \epsilon(w), \epsilon(w))\right) \geq 0$ (Lem. 1 in [66]) and since $\int_{0}^{T}\left(k * \epsilon\left(e_{u}\right), \epsilon(w)\right) \leq\|k\|_{L^{\infty}(0, T)} T\left\|\epsilon\left(e_{u}\right)\right\|_{L^{2}\left(L^{2}\right)}\|\epsilon(w)\|_{L^{2}\left(L^{2}\right)}$, there exists a constant $C_{d}$ independent of $(w, r)$ and $e_{p}$ such that

$$
\rho\left\|\frac{\partial w}{\partial t}\right\|_{L^{2}\left(H^{-1}\right)}+\rho\|w(0)\|_{L^{2}(D)}+2 \eta_{s}\|\epsilon(w)\|_{L^{2}\left(L^{2}\right)}+2\|r\|_{L^{2}\left(L^{2}\right)} \leq C_{d}\left\|e_{p}\right\|_{L^{2}\left(L^{2}\right)} .
$$

Thus, choosing $\bar{v} \equiv 0, \bar{r}=e_{p}$ in (B.3) and after time integration we have

$$
\left\|e_{p}\right\|_{L^{2}\left(L^{2}\right)}^{2}=\int_{0}^{T}\left(e_{p}, \nabla \cdot w\right)
$$

so that equations $(2.15),(3.9)$ lead to

$$
\begin{aligned}
\left\|e_{p}\right\|_{L^{2}\left(L^{2}\right)}^{2}= & 2 \eta_{s} \int_{0}^{T}\left(\epsilon\left(u_{h}\right), \epsilon(\Pi w)\right)+2 \eta_{s} \int_{0}^{T} k *\left(\epsilon\left(u_{h}\right), \epsilon(\Pi w)\right)-\int_{0}^{T}\left(p_{h}, \nabla \cdot \Pi w\right)+\left(\frac{\eta_{p}}{\lambda} f_{2}+g, \epsilon(\Pi w)\right) \\
& +\int_{0}^{T}\left(\rho \frac{\partial u_{h}}{\partial t}-f, \Pi w\right)+\int_{0}^{T}\left(\rho \frac{\partial e_{u}}{\partial t}, w\right)+2 \eta_{s} \int_{0}^{T}\left(\epsilon\left(e_{u}\right), \epsilon(w)\right)+2 \int_{0}^{T}\left(k * \epsilon\left(e_{u}\right), \epsilon(w)\right) \\
& -\int_{0}^{T}\left(i_{h} g-g, \epsilon\left(i_{h}^{C} w\right)\right)-2 k *\left(i_{h} \epsilon\left(u_{h}-\epsilon\left(u_{h}\right)\right), \epsilon\left(i_{h}^{C} w\right)\right) .
\end{aligned}
$$

where $\Pi w=w-i_{h}^{C} w$ and $i_{h}^{C}$ is the Clément's interpolant [23]. We then integrate by parts on each triangle $K \in \mathcal{T}_{h}$ the first four terms in the right hand side of the above equation, use Cauchy-Schwarz and (B.4) inequalities to obtain

$$
\left\|e_{p}\right\|_{L^{2}\left(L^{2}\right)}^{2} \leq C \sum_{K \in \mathcal{T}_{h}} \mu_{K}^{2}\left(f_{1}, u_{0}, f_{2}, w\right)+\int_{0}^{T}\left(\rho \frac{\partial e_{u}}{\partial t}, w\right)+2 \eta_{s} \int_{0}^{T}\left(\epsilon\left(e_{u}\right), \epsilon(w)\right)+2 \int_{0}^{T}\left(k * \epsilon\left(e_{u}\right), \epsilon(w)\right)
$$


where $C$ is a constant only depending on $D$. Integrating by parts the second term in the right hand side, using equation (B.3) and estimate (B.4), we obtain

$$
\left\|e_{p}\right\|_{L^{2}\left(L^{2}\right)}^{2} \leq C \sum_{K \in \mathcal{T}_{h}} \mu_{K}^{2}\left(f_{1}, u_{0}, f_{2}, w\right)+C_{d}\left\|e_{p}\right\|_{L^{2}\left(L^{2}\right)}\left(\left\|\nabla \cdot e_{u}\right\|_{L^{2}\left(L^{2}\right)}+\left\|e_{u}(0)\right\|_{L^{2}(D)}^{2}\right) .
$$

Since $\nabla \cdot u=0$, a Young inequality implies

$$
\left\|e_{p}\right\|_{L^{2}\left(L^{2}\right)}^{2} \leq C \sum_{K \in \mathcal{T}_{h}} \mu_{K}^{2}\left(f_{1}, u_{0}, f_{2}, w\right)
$$

The above estimate coupled with (B.2) leads to

$$
\left\|\epsilon\left(e_{u}\right)\right\|_{L^{2}\left(L^{2}\right)}^{2} \leq C \sum_{K \in \mathcal{T}_{h}} \mu_{K}^{2}\left(f_{1}, u_{0}, f_{2}, w\right)
$$

where $C$ is a constant independent of $\left(f_{1}, u_{0}, f_{2}, w\right),\left(\tilde{u}, \tilde{q}^{D}, \tilde{p}\right)$ and $\left(\tilde{u}_{h}, \tilde{q}_{h}^{D}, \tilde{p}_{h}\right)$.

It remains to prove the a posteriori estimate for $e_{q}=\tilde{q}^{D}-\tilde{q}_{h}^{D}$ where $\tilde{q}^{D}$ satisfies (2.11) and $\tilde{q}_{h}^{D}$ satisfies (3.6). We have

$$
\left(\frac{\partial e_{q}}{\partial t}, e_{q}\right)+\frac{1}{2 \lambda}\left(e_{q}, e_{q}\right)=-\left(\frac{\partial \tilde{q}_{h}^{D}}{\partial t}+\frac{1}{2 \lambda} e_{q}-\left(\nabla \tilde{u}_{h}\right) q^{S}, e_{q}\right)+\left(\nabla\left(\tilde{u}_{h}-\tilde{u}\right), e_{q}\right)
$$

Time integration, Cauchy-Schwarz inequalities and estimate (B.5) conclude the proof.

\section{Appendix C. Regularity of the Ornstein-Uhlenbeck Process}

Let $a \geq 0, b \neq 0, c$ be three constants and $T>0$ be the final time. In this appendix, it will be proved that given $W$ the Brownian motion and $q_{0} \sim \mathcal{N}\left(0, c^{2}\right)$ an initial condition independent of $W$, the solution $q: \Omega \times(0, T) \rightarrow \mathbb{R}$ of

satisfies

$$
d q=-a q d t+b d W, \quad q(0)=q_{0}
$$

$$
q \in L^{\gamma}\left(\Omega ; h^{\mu}([0, T] ; \mathbb{R})\right)
$$

for all $1<\gamma<\infty$ and $0<\mu<1 / 2$. The proof proposed in this appendix follows the ideas of Theorem 5.20 in [24], where the case of linear stochastic equations in infinite dimensions is treated.

We first note that, in order to prove (C.2), it suffices to show that

$$
q \in \mathcal{C}^{1 / 2}\left([0, T] ; L^{\gamma}(\Omega)\right)
$$

for all $1<\gamma<\infty$. Indeed, using the Kolmogorov criterion (see for instance [63], Thm. 2.1), we know that if $q$ satisfies (C.3) then we have

$$
q \in L^{\gamma}\left(\Omega ; \mathcal{C}^{1 / 2-1 / \gamma-\epsilon}([0, T])\right)
$$

for all $1<\gamma<\infty$ and $0<\epsilon \leq 1 / 2-1 / \gamma$. Since for $0<\mu^{\prime}<\mu<1$ we have that $h^{\mu}([0, T]) \subset \mathcal{C}^{\mu^{\prime}}([0, T])$ (see [54]), thus we find that if $q$ satisfies (C.3), then $q$ satisfies (C.2) for all $1<\gamma<\infty, 0<\mu<1 / 2$.

We now prove (C.3). Let us recall that

$$
\|q\|_{C^{1 / 2}\left(L^{\gamma}\right)}=\sup _{t \in[0, T]}\|q(t)\|_{L^{\gamma}}+\sup _{\substack{t, s \in[0, T] \\ t \neq s}} \frac{\|q(t)-q(s)\|_{L^{\gamma}}}{|t-s|^{1 / 2}}
$$


Let $1<\gamma<\infty$. Consider the first term in the right hand side of the above equation. From [42], we know that the solution $q$ of (C.1) satisfies for $t, s \in[0, T]$

$$
\mathbb{E}(q(t))=0, \quad \operatorname{Cov}(q(t), q(s))=\left(c^{2}+\frac{b^{2}}{2 a}\left(\mathrm{e}^{2 a \min (t, s)}-1\right)\right) \mathrm{e}^{-a(t+s)},
$$

when $a>0$ (note that $\mathbb{E}(q(t))=0$, $\operatorname{Cov}(q(t), q(s))=c^{2}+b^{2} \min (t, s)$ when $a=0$ ). Let $m \geq 1$ be an integer such that $\gamma \leq 2 m$. By using Hölder's inequality and since $\mathcal{P}(\Omega)=1$, it follows

$$
\|q(t)\|_{L^{\gamma}} \leq\|q(t)\|_{L^{2 m}}
$$

Since $q$ is a Gaussian process, using integrations by parts there exists a constant $C$ only depending on $\gamma, a, b$ and $c$ such that

$$
\|q(t)\|_{L^{\gamma}} \leq C\|q(t)\|_{L^{2}} .
$$

Using (C.4) and since for $x<0,1-e^{x}<|x|$, we obtain for $t \in[0, T]$

$$
\|q(t)\|_{L^{\gamma}} \leq C \sqrt{c^{2}+b^{2} T}
$$

and

$$
\sup _{t \in[0, T]}\|q(t)\|_{L^{\gamma}}<\infty .
$$

The same arguments can be applied to prove that

$$
\sup _{\substack{t, s \in[0, T] \\ t \neq s}} \frac{\|q(t)-q(s)\|_{L^{\gamma}}}{|t-s|^{1 / 2}}<\infty .
$$

Indeed, by using Hölder's inequality it follows that for $t, s \in[0, T]$

$$
\|q(t)-q(s)\|_{L^{\gamma}} \leq\|q(t)-q(s)\|_{L^{2 m}},
$$

where $m \geq 1$ is an integer such that $\gamma \leq 2 m$. Again, $q(t)-q(s)$ is a Gaussian process and we have

$$
\|q(t)-q(s)\|_{L^{\gamma}} \leq C\|q(t)-q(s)\|_{L^{2}}=C\left(\|q(t)\|_{L^{2}}^{2}+\|q(s)\|_{L^{2}}^{2}-2 \operatorname{Cov}(q(t), q(s))\right)^{1 / 2}
$$

where $C$ is a constant only depending on $\gamma, a, b$ and $c$. Using again (C.4) and since $1-\mathrm{e}^{x}<|x|$ for $x<0$, a simple but tedious calculation yields for $t, s \in[0, T]$

$$
\|q(t)-q(s)\|_{L^{\gamma}} \leq C\left(c^{2}\left(\mathrm{e}^{-2 a s}+\mathrm{e}^{-2 a t}\right)+b^{2}\left(1+\mathrm{e}^{-2 a \min (s, t)}\right)\right)^{1 / 2}|t-s|^{1 / 2},
$$

and (C.7) follows. The estimates (C.6) and (C.7) show that $q \in C^{1 / 2}\left([0, T] ; L^{\gamma}(\Omega)\right)$, for all $1<\gamma<\infty$.

\section{REFERENCES}

[1] R. Akhavan and Q. Zhou, A comparison of FENE and FENE-P dumbbell and chain models in turbulent flow. J. Non-Newton. Fluid 109 (2003) 115-155.

[2] N. Arada and A. Sequeira, Strong steady solutions for a generalized Oldroyd-B model with shear-dependent viscosity in a bounded domain. Math. Mod. Meth. Appl. S. 13 (2003) 1303-1323.

[3] F.P.T. Baaijens, Mixed finite element methods for viscoelastic flow analysis: a review. J. Non-Newton. Fluid 79 (1998) $361-385$.

[4] I. Babuška, R. Durán and R. Rodríguez, Analysis of the efficiency of an a posteriori error estimator for linear triangular finite elements. SIAM J. Numer. Anal. 29 (1992) 947-964. 
[5] J. Baranger and H. El Amri, Estimateurs a posteriori d'erreur pour le calcul adaptatif d'écoulements quasi-newtoniens. RAIRO Modél. Math. Anal. Numér. 25 (1991) 931-947.

[6] J. Baranger and D. Sandri, Finite element approximation of viscoelastic fluid flow: existence of approximate solutions and error bounds. I. Discontinuous constraints. Numer. Math. 63 (1992) 13-27.

[7] J. Baranger and S. Wardi, Numerical analysis of a FEM for a transient viscoelastic flow. Comput. Method. Appl. M. 125 (1995) 171-185.

[8] J.W. Barrett, C. Schwab and E. Süli, Existence of global weak solutions for some polymeric flow models. Math. Mod. Meth. Appl. S. 15 (2005) 939-983.

[9] R. Bird, C. Curtiss, R. Armstrong and O. Hassager, Dynamics of polymeric liquids, Vol. 1 and 2. John Wiley \& Sons, New York, 1987.

[10] A. Bonito, Ph. Clément and M. Picasso, Mathematical and numerical analysis of a simplified time-dependent viscoelastic flow. Numer. Math. (submitted).

[11] A. Bonito, Ph. Clément and M. Picasso, Mathematical analysis of a simplified Hookean dumbbells model arising from viscoelastic flows. J. Evol. Equ. 6 (2006) 381-398.

[12] A. Bonito, M. Picasso and M. Laso, Numerical simulation of 3d viscoelastic flows with complex free surfaces. J. Comput. Phys. 215 (2006) 691-716.

[13] J. Bonvin and M. Picasso, Variance reduction methods for connffessit-like simulations. J. Non-Newton. Fluid 84 (1999) 191215 .

[14] J. Bonvin and M. Picasso, A finite element/Monte-Carlo method for polymer dilute solutions. Comput. Vis. Sci. 4 (2001) 93-98. Second AMIF International Conference (Il Ciocco, 2000).

[15] J. Bonvin, M. Picasso and R. Stenberg, GLS and EVSS methods for a three-field Stokes problem arising from viscoelastic flows. Comput. Method. Appl. M. 190 (2001) 3893-3914.

[16] B.H.A.A. van den Brule, A.P.G. van Heel and M.A. Hulsen, Simulation of viscoelastic flows using Brownian configuration fields. J. Non-Newton. Fluid 70 (1997) 79-101.

[17] B.H.A.A. van den Brule, A.P.G. van Heel and M.A. Hulsen, On the selection of parameters in the FENE-P model. J. NonNewton. Fluid 75 (1998) 253-271.

[18] B.H.A.A van den Brule, M.A. Hulsen and H.C. Öttinger, Brownian configuration fields and variance reduced connffessit. J. Non-Newton. Fluid 70 (1997) 255-261.

[19] B. Buffoni and J. Toland, Analytic theory of global bifurcation. Princeton Series in Applied Mathematics. Princeton University Press, Princeton, NJ, (2003).

[20] G. Caloz and J. Rappaz, Numerical analysis for nonlinear and bifurcation problems, in Handbook of numerical analysis, Vol. V, North-Holland, Amsterdam (1997) 487-637.

[21] C. Chauvière and A. Lozinski, A fast solver for Fokker-Planck equation applied to viscoelastic flows calculations: 2D FENE model. J. Comput. Phys. 189 (2003) 607-625.

[22] P.G. Ciarlet and J.-L. Lions, editors, Handbook of numerical analysis. Vol. II. North-Holland, Amsterdam, (1991). Finite element methods. Part 1.

[23] P. Clément, Approximation by finite element functions using local regularization. RAIRO Anal. Numér. 9 (1975) 77-84.

[24] G. Da Prato and J. Zabczyk, Stochastic equations in infinite dimensions, Vol. 44 of Encyclopedia of Mathematics and its Applications. Cambridge University Press, Cambridge (1992).

[25] W. E, T. Li and P. Zhang, Convergence of a stochastic method for the modeling of polymeric fluids. Acta Math. Sin. 18 (2002) $529-536$

[26] W. E, T. Li and P. Zhang, Well-posedness for the dumbbell model of polymeric fluids. Comm. Math. Phys. 248 (2004) $409-427$.

[27] V.J. Ervin and N. Heuer, Approximation of time-dependent, viscoelastic fluid flow: Crank-Nicolson, finite element approximation. Numer. Methods Partial Differ. Equ. 20 (2004) 248-283.

[28] V.J. Ervin and W.W. Miles, Approximation of time-dependent viscoelastic fluid flow: SUPG approximation. SIAM J. Numer. Anal. 41 (2003) 457-486 (electronic).

[29] X. Fan, Molecular models and flow calculation: I. the numerical solutions to multibead-rod models in inhomogeneous flows. Acta Mech. Sin. 5 (1989) 49-59.

[30] X. Fan, Molecular models and flow calculation: II. simulation of steady planar flow. Acta Mech. Sin. 5 (1989) $216-226$.

[31] M. Farhloul and A.M. Zine, A new mixed finite element method for viscoelastic fluid flows. Int. J. Pure Appl. Math. 7 (2003) 93-115.

[32] E. Fernández-Cara, F. Guillén and R.R. Ortega, Mathematical modeling and analysis of viscoelastic fluids of the Oldroyd kind, in Handbook of numerical analysis, Vol. VIII, North-Holland, Amsterdam (2002) 543-661.

[33] A. Fortin, R. Guénette and R. Pierre, On the discrete EVSS method. Comput. Method. Appl. M. 189 (2000) $121-139$.

[34] M. Fortin and R. Pierre, On the convergence of the mixed method of Crochet and Marchal for viscoelastic flows. Comput. Method. Appl. M. 73 (1989) 341-350.

[35] Y. Giga, Analyticity of the semigroup generated by the Stokes operator in $L_{r}$ spaces. Math. Z. 178 (1981) $297-329$.

[36] E. Grande, M. Laso and M. Picasso, Calculation of variable-topology free surface flows using CONNFFESSIT. J. Non-Newton. Fluid 113 (2003) 127-145. 
[37] C. Guillopé and J.-C. Saut, Existence results for the flow of viscoelastic fluids with a differential constitutive law. Nonlinear Anal-theor. 15 (1990) 849-869.

[38] B. Jourdain, T. Lelièvre and C. Le Bris, Numerical analysis of micro-macro simulations of polymeric fluid flows: a simple case. Math. Mod. Meth. Appl. S. 12 (2002) 1205-1243.

[39] B. Jourdain, C. Le Bris and T. Lelièvre, On a variance reduction technique for micro-macro simulations of polymeric fluids. J. Non-Newton. Fluid 122 (2004) 91-106.

[40] B. Jourdain, T. Lelièvre and C. Le Bris, Existence of solution for a micro-macro model of polymeric fluid: the FENE model. J. Funct. Anal. 209 (2004) 162-193.

[41] B. Jourdain, C. Le Bris, T. Lelièvre and F. Otto, Long-time asymptotics of a multiscale model for polymeric fluid flows. Arch. Ration. Mech. An. 181 (2006) 97-148.

[42] I. Karatzas and S.E. Shreve, Brownian motion and stochastic calculus, volume 113 of Graduate Texts in Mathematics. SpringerVerlag, New York (1991).

[43] R. Keunings, On the Peterlin approximation for finitely extensible dumbbells. J. Non-Newton. Fluid 68 (1997) 85-100.

[44] R. Keunings, Micro-marco methods for the multi-scale simulation of viscoelastic flow using molecular models of kinetic theory, in Rheology Reviews, D.M. Binding, K. Walters (Eds.), British Society of Rheology (2004) 67-98.

[45] S. Larsson, V. Thomée and L.B. Wahlbin, Numerical solution of parabolic integro-differential equations by the discontinuous Galerkin method. Math. Comp. 67 (1998) 45-71.

[46] M. Laso and H.C. Öttinger, Calculation of viscoelastic flow using molecular models: the connffessit approach. J. Non-Newton. Fluid 47 (1993) 1-20.

[47] M. Laso, H.C. Öttinger and M. Picasso, 2-d time-dependent viscoelastic flow calculations using connffessit. AICHE Journal 43 (1997) 877-892.

[48] C. Le Bris and P.-L. Lions, Renormalized solutions of some transport equations with partially $W^{1,1}$ velocities and applications. Ann. Mat. Pur. Appl. 183 (2004) 97-130.

[49] T. Lelièvre, Optimal error estimate for the CONNFFESSIT approach in a simple case. Comput. Fluids 33 (2004) 815-820.

[50] T. Li and P. Zhang, Convergence analysis of BCF method for Hookean dumbbell model with finite difference scheme. Multiscale Model. Simul. 5 (2006) 205-234.

[51] T. Li, H. Zhang and P. Zhang, Local existence for the dumbbell model of polymeric fluids. Comm. Partial Diff. Eq. 29 (2004) 903-923.

[52] P.L. Lions and N. Masmoudi, Global solutions for some Oldroyd models of non-Newtonian flows. Chinese Ann. Math. Ser. B 21 (2000) 131-146.

[53] A. Lozinski and R.G. Owens, An energy estimate for the Oldroyd B model: theory and applications. J. Non-Newton. Fluid 112 (2003) 161-176.

[54] A. Lunardi, Analytic semigroups and optimal regularity in parabolic problems. Progress in Nonlinear Differential Equations and their Applications, 16 Birkhäuser Verlag, Basel (1995).

[55] A. Machmoum and D. Esselaoui, Finite element approximation of viscoelastic fluid flow using characteristics method. Comput. Method. Appl. M. 190 (2001) 5603-5618.

[56] K. Najib and D. Sandri, On a decoupled algorithm for solving a finite element problem for the approximation of viscoelastic fluid flow. Numer. Math. 72 (1995) 223-238.

[57] H.C. Öttinger, Stochastic processes in polymeric fluids. Springer-Verlag, Berlin (1996).

[58] R.G. Owens and T.N. Phillips, Computational rheology. Imperial College Press, London (2002).

[59] M. Picasso and J. Rappaz, Existence, a priori and a posteriori error estimates for a nonlinear three-field problem arising from Oldroyd-B viscoelastic flows. ESAIM: M2AN 35 (2001) 879-897.

[60] A. Quarteroni and A. Valli, Numerical Approximation of Partial Differential Equations. Number 23 in Springer Series in Computational Mathematics. Springer-Verlag (1991).

[61] M. Renardy, Existence of slow steady flows of viscoelastic fluids of integral type. Z. Angew. Math. Mech. 68 (1988) T40-T44.

[62] M. Renardy, An existence theorem for model equations resulting from kinetic theories of polymer solutions. SIAM J. Math. Anal. 22 (1991) 313-327.

[63] D. Revuz and M. Yor, Continuous martingales and Brownian motion, of Grundlehren der Mathematischen Wissenschaften [Fundamental Principles of Mathematical Sciences]. 293 Springer-Verlag, Berlin (1994).

[64] D. Sandri, Analyse d'une formulation à trois champs du problème de Stokes. RAIRO Modél. Math. Anal. Numér. 27 (1993) $817-841$.

[65] D. Sandri, Finite element approximation of viscoelastic fluid flow: existence of approximate solutions and error bounds. Continuous approximation of the stress. SIAM J. Numer. Anal. 31 (1994) 362-377.

[66] P.E. Sobolevskiu, Coerciveness inequalities for abstract parabolic equations. Dokl. Akad. Nauk SSSR 157 (1964) 52-55.

[67] R. Verfürth, A posteriori error estimators for the Stokes equations. Numer. Math. 55 (1989) $309-325$.

[68] T. von Petersdorff and Ch. Schwab, Numerical solution of parabolic equations in high dimensions. ESAIM: M2AN 38 (2004) 93-127.

[69] H. Zhang and P. Zhang, Local existence for the FENE-Dumbbells model of polymeric liquids. Arch. Ration. Mech. An. 181 (2006) 373-400. 